A - Research concept and design

B - Collection and/or assembly of data

C - Data analysis and interpretation

D - Writing the article

E - Critical revision of the article

F - Final approval of article

Received: 2021-12-28

Accepted: 2022-01-31

Published: 2022-02-01

\section{Efficacy of biofeedback in rehabilitation of musculoskeletal disorders: A systematic review}

\author{
Hernán Andrés de la Barra Ortiz ${ }^{\mathrm{A}, \mathrm{C}-\mathrm{F} *} \mathbb{D}$, Alexandra Meza \\ Matamala ${ }^{A-C, E-F} \mathbb{D}$, Francisca López Inostroza ${ }^{B-F} \mathbb{D}$, \\ Catalina Lobos Araya ${ }^{\mathrm{B}-\mathrm{D}, \mathrm{F}}$ (D), Valeria Núñez Mondaca ${ }^{\mathrm{B}-\mathrm{D}, \mathrm{F}}$ (ii)
}

Exercise and Rehabilitation Sciences Laboratory, Faculty of Rehabilitation Sciences, School of Physical Therapy, Universidad Andres Bello, Chile

\title{
Abstract
}

Musculoskeletal disorders (MSD) are a frequent reason for consultation and the main cause of disability in population. Electromyographic biofeedback or myofeedback (MF) is a promising treatment in rehabilitation, although studies supporting its benefits in MSD have declined in recent years. The objective of this review was to describe the efficacy of MF in function recovery, strength increase and muscle relaxation in MSD. Randomized clinical trials (RCTs) were identified in Pubmed, Scopus, Web of Science, Cinahl and Science Direct databases dated September 2, 2021. Four independent researchers reviewed articles titles and abstracts to determine their eligibility. Risk of bias and articles quality was assessed using Rob2 tool (Cochrane) and PEDro scale. Functionality improvement, strength increase, and muscle relaxation were considered as main outcome. Search strategy yielded 160 articles after eliminating duplicates, reducing to 26 when selection criteria were applied. Articles were classified in strengthening $(n=16)$ and muscle relaxation $(n=10)$ according to MF therapeutic aim. Eighteen articles were rated as low risk of bias (69.22\%) and an average internal validity of 6 points was obtained. Studies showed improvements in functionality, strength increase and pain reduction with statistical significance when MF were complemented with therapeutic exercises or other physical agents modalities $(p<0.005)$. MF also showed a decrease in fear of movement, depression, and pain perception, suggesting central modulating effects. This review supports MF efficacy in MSD rehabilitation, showing improvements in functionality and pain reduction. The review allowed to establish a dosage recommendation based on articles analysis which can be considered for future RCTs.

\section{Keywords: rehabilitation, biofeedback, musculoskeletal diseases, myofeedback, recovery of function}

\section{Introduction}

Musculoskeletal pain (MSP) is currently recognized as one of the main reasons for medical consultation and the most common cause of disability in adults worldwide, affecting about $47 \%$ of the population $[1,2]$. MSP is classified chronologically as acute or chronic depending on whether it persists for more than three months, which occurs in 39 to $45 \%$ of patients [1,3]. Chronic MSP is associated with sleep disturbances, fatigue, depression, and activity limitations, which affect life quality and work productivity [4,5]. Currently, its prevalence in adults is $20 \%$, and it is estimated that, by 2050 , it will reach $50 \%$ [3]. 
Although MSP is usually of nociceptive origin, people with chronic disorders may experience neuropathic pain (NP) or nociplastic pain (NCP) [6,7]. NP occurs due to injury or dysfunction of the nervous system, manifesting clinically with hyperalgesia and allodynia, muscle weakness, and impaired reflexes [8]. NCP is characterized by altered nociception phenomena in which there is no clear disease evidence, real tissue damage or somatosensory system injury, causing fibromyalgia, regional pain complex, and nonspecific lumbar pain $[7,8]$. The transition from acute or chronic MSP to NCP is associated with central sensitization phenomena produced by changes in ion channels expression of nociceptive neurons, which triggers their greater excitability, reinforced synaptic transmission, and decreased pain inhibition processes at the spinal cord posterior horn, subcortical and cortical areas such as the thalamus, somatosensory cortex, or primary motor cortex $[5,9,10]$. Furthermore, less neural connectivity between the prefrontal cortex and somatosensory areas has been documented in patients with chronic MSP, along with more synaptic networks with the insula, which exacerbates the psychological and emotional components $[11,12]$.

Currently, more than 150 musculoskeletal disorders (MSD) have been recognized, highlighting degenerative and autoimmune joint diseases, fractures, dislocations, muscle injuries, sprains, and tendinopathies $[1,2,6]$. Most frequent presentations include the back, neck, shoulders, and knees, although multiregional MSDs are also described $[1,13]$. MSD can debut at any age; however, their prevalence increases from adolescence onwards [14].

MSD medical treatment has been oriented to their clinical symptoms with analgesic drugs, non-steroidal anti-inflammatory drugs (NSAIDs), opiates or corticosteroids infiltration, which produce symptoms relief, although they aren't a functional improvement guarantee, added to the fact that for some conditions their efficacy and safety are uncertain $[15,16]$. In addition, the overuse of drugs has been associated with analgesic tolerance, hyperalgesia, and adverse effects, such as nausea, vomiting, gastrointestinal irritation, arterial hypertension, kidney problems, or hepatotoxicity [15-17]. Surgery is an alternative for many MSD with refractory symptoms or pharmacological treatment resistance, although its efficacy is dependent on clinical conditions, personal and contextual variables, added to patient economic costs $[15,18]$.

Physical therapy is a non-pharmacological option for pain management and functional recovery in MSD, using treatments such as therapeutic ultrasound (US), transcutaneous electrical nerve stimulation (TENS), photobiomodulation (PBM), manual therapy, and therapeutic exercise [19-21]. Another therapeutic alternative is electromyographic biofeedback or myofeedback (MF), a safe and non-invasive intervention supported for strength increase, muscle relaxation, and functional recovery in a variety of MSD [22-25] being used for motor reeducation and muscle training in musculoskeletal, neurological, and pelvic floor conditions [26,27]. MF captures motor neuron's myoelectric signals through surface electrodes (extracellular technique) or percutaneous (intracellular technique), converting them into visual or auditory information that provides patient immediate feedback on voluntary muscle contraction (VMC) or muscle activity at rest. MF electrodes perceive voltage changes in microvolts associated with neuromuscular activity, transmitting them to an amplifier and processor that filters, integrates, and rectifies bioelectric waves, transforming them into digital signals represented graphically or audibly. This information is used to promote or decrease motor activity depending on whether the objective is to strengthen, relax or re-educate motor patterns [28].

Biological feedback processes are essential for human movement realization and regulation. Motor neurons activity is the result of the interaction between the central nervous system (CNS) and sensory function, through an intrinsic feedback system formed by neurological circuits between the motor system, proprioceptors, joint receptors, skin receptors, vestibular system, and visual system [28-30]. On the other hand, visual and/or auditory biofeedback favors the different levels of afferent and efferent integration in CNS, allowing the patient to control motor activity [28].

Even though the literature describes MF as a valuable therapeutic resource, publications that support its use for MSD have decreased in recent years, showing greater development in neurological and pelvic floor rehabilitation, so an update in musculoskeletal rehabilitation is necessary. Thus, the objective of this systematic review (SR) was to describe the efficacy of MF in function recovery, strength increase and muscle relaxation in MSD.

\section{Materials and methods}

\section{Study design}

This SR adheres to the PRISMA statement on the reporting of preference items for systematic reviews and meta-analysis (available at http://www.prismastatement.org) [31]. The research was uploaded electronically to the International SR Prospective Registry (PROSPERO) of the National Institute for Health Research (NIHR), obtaining the identification code CRD42021228046. 
The PICO acronym (participants, intervention, comparison, and outcome) was used to structure the research question and search algorithm based on the following elements: patients with MSD, intervened with MF, compared with a control, sham application or placebo, and evaluating, as main outcome, changes in functionality and, as secondary result, pain decrease, range of motion (ROM) increase, or muscle strength.

\section{Search strategy}

The SR was performed considering PubMed, Scopus, Web of Science (WoS), Cinahl, and Science Direct electronic databases with the last update on September 2, 2021. For the search, keywords from the MeSH dictionary were chosen (Medical Subject Headings, https:// www.ncbi.nlm.nih.gov/mesh). Search terms included "Biofeedback", "Myofeedback", "Myoelectric biofeedback", "Rehabilitation", "Recovery of Function" and "Musculoskeletal Diseases" connected through the boolean terms "OR" and "AND" obtaining the following algorithm: (((("Biofeedback") OR ("Myofeedback")) OR ("Myoelectric biofeedback")) AND (("Rehabilitation") OR ("Recovery of Function"))) AND ((“Musculoskeletal Diseases") OR ("Musculoskeletal Pain")).

Searches for each database were downloaded (nbib, ris or ciw formats) and the files were analyzed with the Rayyan tool developed for the preliminary selection of abstracts and article titles (https://www.rayyan.ai) [32]. Four independent researchers (AM, FL, CL, VN) analyzed articles titles and abstracts based on the selection criteria, classifying them in the "included", "maybe" and "excluded" categories. In addition, the references of the studies were examined and revised in terms of their country of origin, author, affiliated institutions, and enrollment periods to identify and exclude duplicate publications. Articles in the "maybe" category were reviewed by the research team to determine their inclusion in the final count. Articles with incomplete abstracts were discarded from the analysis, and each investigator recorded their reasons for exclusion.

For included articles, study objective, PEDro scale score, participants demographic data, follow-up period, evaluation time, treatment protocol with MF and results in the interest outcomes were analyzed [33].

\section{Selection criteria}

Inclusion criteria considered: (1) randomized clinical trials (RCT), (2) human studies, (3) articles in English or Spanish, (4) participants older than 18 years, (5) participants with MSD, (6), studies that used MF alone or with another intervention in MSD rehabilitation, (7) comparison with another treatment, sham, or placebo, (7) outcome measures including changes in function, muscle strength, muscle relaxation, pain, or range of motion. The exclusion criteria were: (i) case report studies, systematic reviews (SR), meta-analysis (MT) and literature reviews, (ii) animal-testing or in vitro studies, (iii) use of MF in non-musculoskeletal conditions, and (iv) studies with incomplete abstracts or texts.

\section{Article's quality and risk of bias}

The articles' internal validity was determined with the PEDro scale [33]. Each researcher performed an independent assessment, and any disagreement was subsequently discussed to establish consensus. RCTs with scores less than five were classified as "low quality," while scores greater than or equal to 5 were considered "high quality."

Risk of bias was assessed using the RoB.2 tool (Cochrane Collaboration tool for RCT analysis in SR) for the following domains [34]; (1) bias arising from randomization process, (2) bias due to deviations from planned interventions, (3) bias due to missing outcome data, (4) outcome measurements bias, (5) bias in reported outcome selection, and (6) overall article bias. The investigators rated the risk of bias for each criterion as high, low, unclear, or no information in case the data provided were not sufficient to decide. Box and summary plots were constructed with the Robvis tool [35]. Studies with two or more high risks of bias were considered as low quality [34].

\section{Results}

\section{Search results}

Preliminary search strategy yielded a total of 5,141 articles for selected databases (Pubmed, $\mathrm{n}=25$; Scopus, $\mathrm{n}=2918 ;$ WoS, $\mathrm{n}=10$; Cinahl, $\mathrm{n}=1494$, and Science Direct, $\mathrm{n}=695$ ). After reviewing titles and abstracts, 160 articles were classified as "possible" and "included" when applying selection criteria. The researchers reached consensus on these articles, discarding 134 studies, and finally including 26 for analysis [22-25,35-55]. The main reasons for exclusion were surface electromyography studies, other types of studies, articles in another language, studies that addressed non-musculoskeletal conditions, and articles with incomplete or unavailable abstracts. Figure 1 shows the PRISMA flow chart with a summary of the screening results [57].

\section{Risk of bias and quality}

This SR rated $7.69 \%$ of articles $(n=2)$ as high risk of bias [23,25,27,33,36,38,43,45], especially in domains 1 and 2 for RoB.2 tool $[56,57]$. On the other hand, $26.92 \%(\mathrm{n}=7)$ did not present risks of bias for any of domains $[22,24,26,28,35,37,42,44]$, while $42.30 \%$ presented at least some concern, especially for 


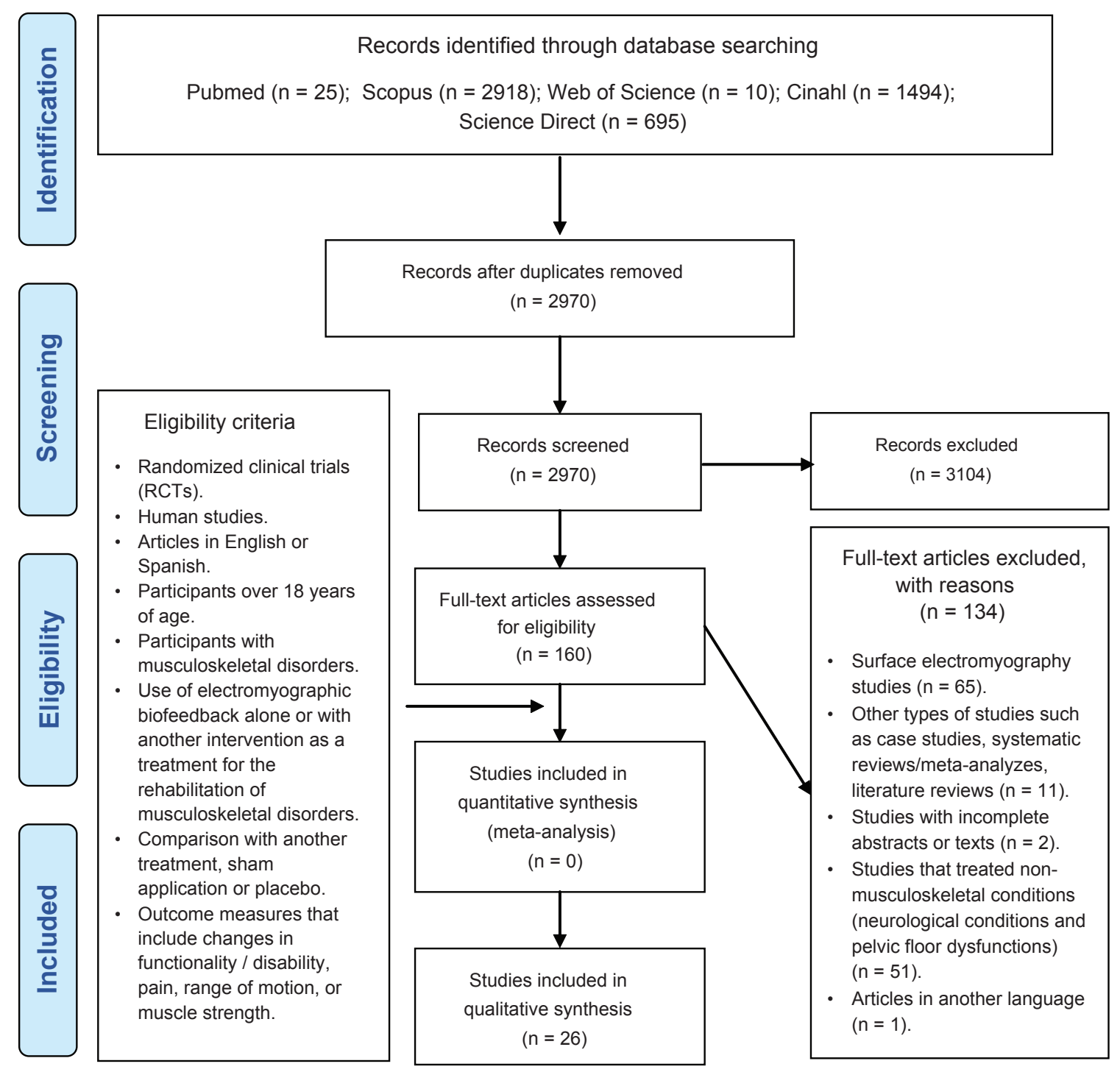

Fig. 1. Flowchart of included studies in accordance with PRISMA guidelines

random assignment. Figure 2 summarizes risk of bias of the selected articles.

Table 1 shows the PEDro score for the 26 RCT. Internal validity shows that $65.38 \%$ of articles $(n=17)$ are of high quality (score greater than or equal to 5 for PEDro scale) with an average of 7 points for all the articles [33].

\section{Study characteristics}

Studies were grouped in Tables 2 and 3 according to their MF therapeutic aim (strengthening or muscle relaxation), summarizing the characteristics of $26 \mathrm{RCT}$ in study groups, evaluation sessions, treatment sessions and the outcome measures of the variables of interest. Table 2 shows that 16 articles (61.53\%) report MF for muscle strengthening, while table 3 shows 10 studies for muscle relaxation $(38.46 \%)$. Strengthening MF studies included post-arthroscopic meniscal injury $(\mathrm{n}=4,25.00 \%)$, subacromial impingement syndrome
(SAIS) $(\mathrm{n}=2,12.5 \%)$, patellofemoral pain syndrome (PFPS) $(n=2,12.5 \%)$, anterior cruciate ligament reconstruction (ACLR) $(\mathrm{n}=2,12.5 \%)$, knee osteoarthritis $(O A)(n=5,31.25 \%)$, non-surgical meniscal injuries $(\mathrm{n}=3,18.75 \%)$ and nucleus hernia pulposus $(\mathrm{n}=1$, $6.25 \%$ ) [22,23,35-48]. On the other hand, MF muscle relaxation studies included cervicobrachialgia $(\mathrm{n}=3$, $30.00 \%)$, neck pain $(\mathrm{n}=2,20.00 \%)$, whiplash syndrome (WPS) $(\mathrm{n}=2,20.00 \%)$, cervical radiculopathy $(\mathrm{n}=1$, $10.00 \%)$, fibromyalgia $(\mathrm{n}=1,10.00 \%)$, low back pain syndrome (LBPS) $(\mathrm{n}=1,10.00 \%)[24,25,49-56]$.

Six studies (23.07\%) used MF in experimental groups (EG) without other added treatment [41,50,51,54-56], while the remaining ones applied MF combined with another treatment. Isolated MF applications were mainly aimed at achieving muscle relaxation in neck pain, cervicobrachialgia, and LBPS [50,51,55,56], while only Choi (2015) applied isolated MF for strengthening in knee OA [41]. 


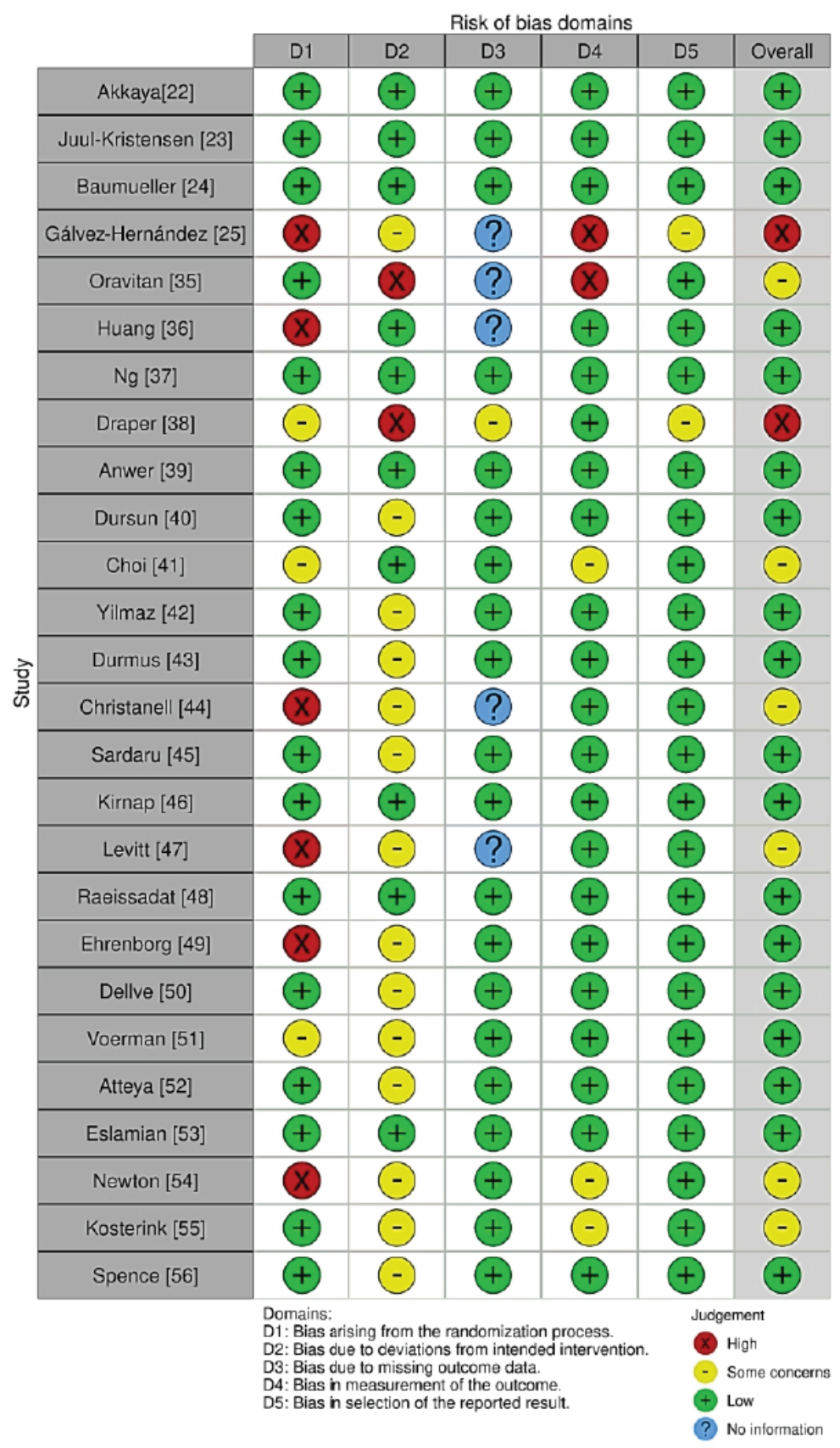

Bias arising from the randomization process Bias due to deviations from intended interventions Bias due to missing outcome data Bias in measurement of the outcome Bias in selection of the reported result

Overall risk of bias

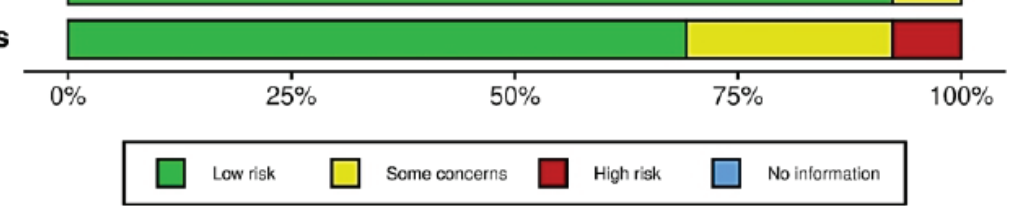

Fig. 2. Risk of bias for the selected studies (ROBVIS tool) 
Tab. 1. PEDro scale scores of analyzed studies

\begin{tabular}{|c|c|c|c|c|c|c|c|c|c|c|c|c|c|}
\hline \multirow{2}{*}{$\begin{array}{c}\text { Clinical } \\
\text { trial } \\
\text { number }\end{array}$} & \multirow[b]{2}{*}{ Author, year of publication } & \multicolumn{11}{|c|}{ PEDro scale criteria } & \multirow{2}{*}{$\begin{array}{l}\text { Total } \\
\text { score }\end{array}$} \\
\hline & & 1 & 2 & 3 & 4 & 5 & 6 & 7 & 8 & 9 & 10 & 11 & \\
\hline 1 & Akkaya, 2012 [22] & 0 & 1 & 1 & 1 & 0 & 0 & 1 & 1 & 0 & 1 & 1 & 7 \\
\hline 2 & Juul-Kristensen, 2019 [23] & 1 & 1 & 1 & 1 & 0 & 0 & 1 & 1 & 1 & 1 & 1 & 8 \\
\hline 3 & Baumueller, 2007 [24] & 1 & 1 & 0 & 0 & 1 & 0 & 0 & 1 & 0 & 1 & 1 & 5 \\
\hline 4 & Gálvez-Hernández, 2016 [25] & 1 & 0 & 0 & 0 & 0 & 0 & 0 & 0 & 0 & 1 & 0 & 1 \\
\hline 5 & Oravitan, 2013 [35] & 0 & 1 & 0 & 1 & 0 & 0 & 0 & 0 & 0 & 1 & 1 & 4 \\
\hline 6 & Huang, 2013 [36] & 1 & 0 & 0 & 1 & 1 & 0 & 0 & 1 & 0 & 1 & 1 & 5 \\
\hline 7 & Ng. 2008 [37] & 0 & 1 & 0 & 1 & 0 & 0 & 1 & 1 & 0 & 0 & 1 & 5 \\
\hline 8 & Draper, 1997 [38] & 0 & 1 & 0 & 0 & 0 & 0 & 0 & 0 & 0 & 1 & 0 & 2 \\
\hline 9 & Anwer, 2011 [39] & 0 & 1 & 0 & 0 & 1 & 0 & 0 & 0 & 1 & 1 & 1 & 5 \\
\hline 10 & Dursun, 2001 [40] & 1 & 1 & 0 & 1 & 0 & 0 & 0 & 1 & 0 & 1 & 1 & 5 \\
\hline 11 & Choi, 2015 [41] & 1 & 1 & 0 & 1 & 0 & 0 & 0 & 0 & 0 & 1 & 1 & 4 \\
\hline 12 & Yilmaz, 2010 [42] & 1 & 1 & 0 & 1 & 0 & 0 & 0 & 1 & 0 & 1 & 1 & 5 \\
\hline 13 & Durmus, 2007 [43] & 1 & 1 & 0 & 1 & 0 & 0 & 0 & 1 & 0 & 1 & 1 & 5 \\
\hline 14 & Christanell, 2012 [44] & 1 & 0 & 0 & 1 & 0 & 0 & 0 & 1 & 0 & 1 & 1 & 4 \\
\hline 15 & Sardaru, 2018 [45] & 1 & 1 & 0 & 1 & 0 & 0 & 0 & 1 & 1 & 1 & 1 & 6 \\
\hline 16 & Kirnap, 2005 [46] & 0 & 1 & 0 & 1 & 0 & 0 & 0 & 1 & 0 & 1 & 1 & 5 \\
\hline 17 & Levitt, 1995 [47] & 1 & 0 & 0 & 1 & 0 & 0 & 0 & 0 & 0 & 1 & 1 & 3 \\
\hline 18 & Raeissadat, 2018 [48] & 1 & 1 & 0 & 1 & 0 & 0 & 1 & 1 & 1 & 1 & 1 & 7 \\
\hline 19 & Ehrenborg, 2010 [49] & 1 & 0 & 0 & 1 & 0 & 0 & 0 & 1 & 1 & 1 & 1 & 5 \\
\hline 20 & Dellve, 2011 [50] & 1 & 1 & 1 & 1 & 0 & 0 & 0 & 0 & 0 & 1 & 1 & 5 \\
\hline 21 & Voerman [51] & 1 & 1 & 0 & 1 & 0 & 0 & 0 & 1 & 1 & 0 & 1 & 5 \\
\hline 22 & Atteya, 2004 [52] & 1 & 1 & 0 & 1 & 0 & 0 & 0 & 1 & 1 & 0 & 1 & 5 \\
\hline 23 & Eslamian, 2020 [53] & 1 & 1 & 1 & 1 & 0 & 0 & 1 & 1 & 1 & 1 & 1 & 8 \\
\hline 24 & Newton, 1995 [54] & 1 & 0 & 0 & 1 & 0 & 0 & 0 & 0 & 0 & 1 & 1 & 3 \\
\hline 25 & Kosterink, 2010 [55] & 1 & 1 & 0 & 1 & 0 & 0 & 0 & 0 & 0 & 1 & 1 & 4 \\
\hline 26 & Spence, 1995 [56] & 0 & 1 & 0 & 0 & 0 & 0 & 0 & 1 & 0 & 1 & 1 & 4 \\
\hline
\end{tabular}

PEDro (Physiotherapy Evidence Database) scale criteria:

(1) The selection criteria were specified.

(2) Subjects were randomized into groups (in a crossover study, subjects were randomized as they received treatments).

(3) The assignment was hidden.

(4) The groups were similar at the beginning in relation to the most important prognostic indicators.

(5) All subjects were blinded.

(6) All therapists who administered the therapy were blinded.

(7) All assessors who measured at least one key outcome were blinded.

(8) Measures of at least one of the key outcomes were obtained from more than $85 \%$ of the subjects initially assigned to the groups.

(9) Results were presented for all subjects who received treatment or were assigned to the control group, or, when this could not be the case, data for at least one key outcome were analysed by 'intention to treat'.

(10) Results of statistical comparisons between groups were reported for at least one key outcome.

(11) The study provides point and variability measures for at least one key outcome. 


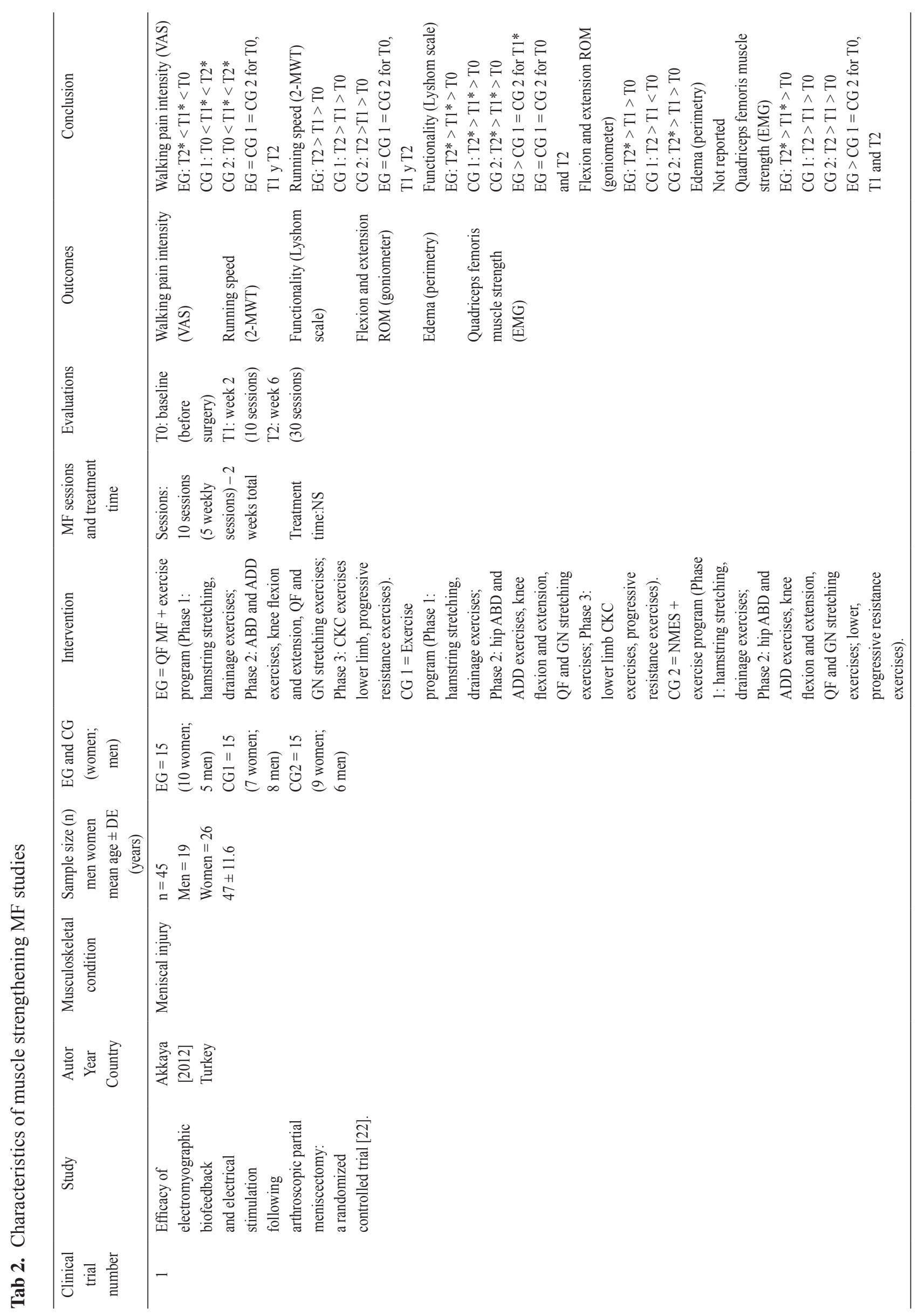




\begin{tabular}{|c|c|c|}
\hline & 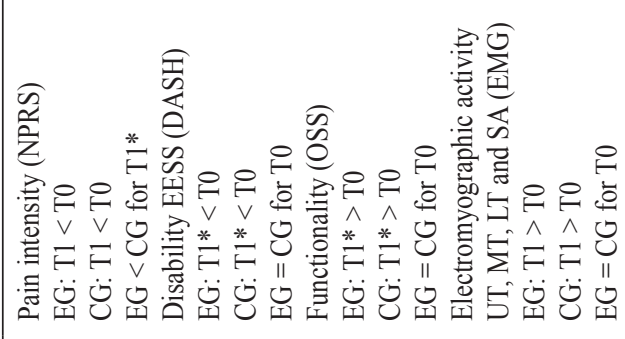 & 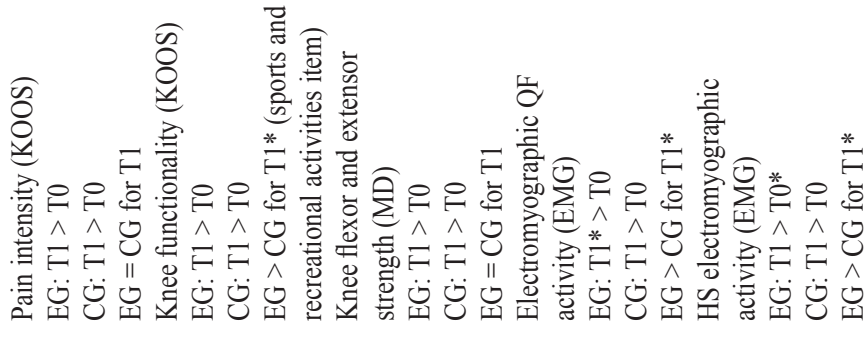 \\
\hline & 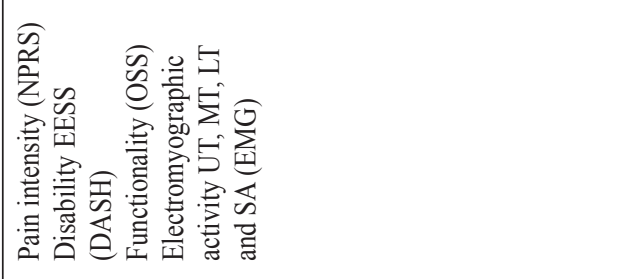 & 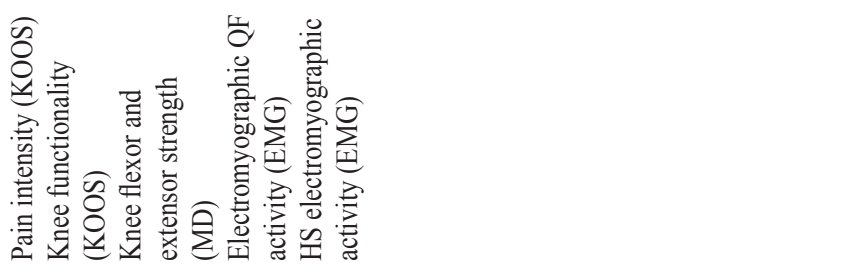 \\
\hline .气 & 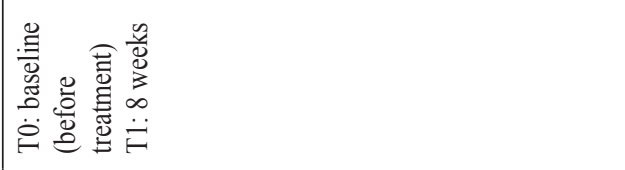 & 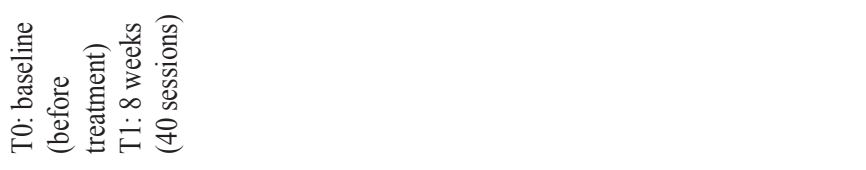 \\
\hline 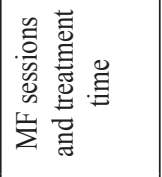 & 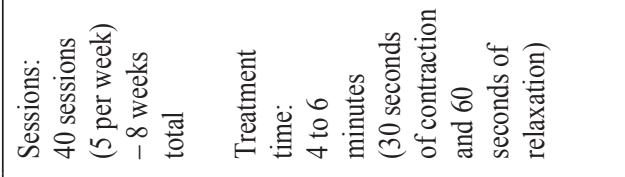 & 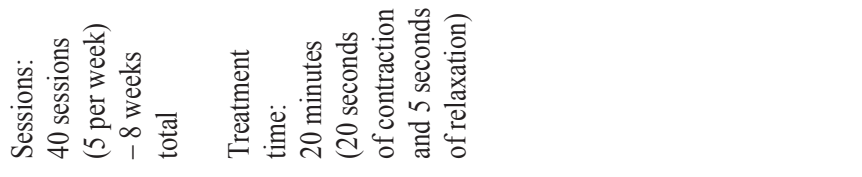 \\
\hline & 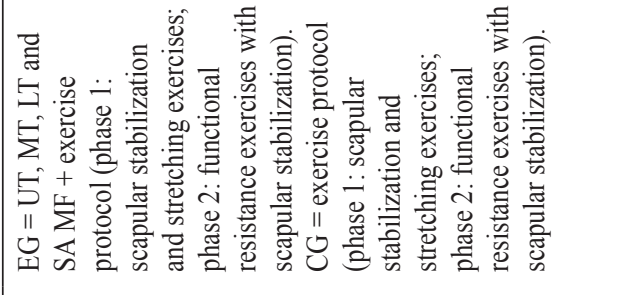 & 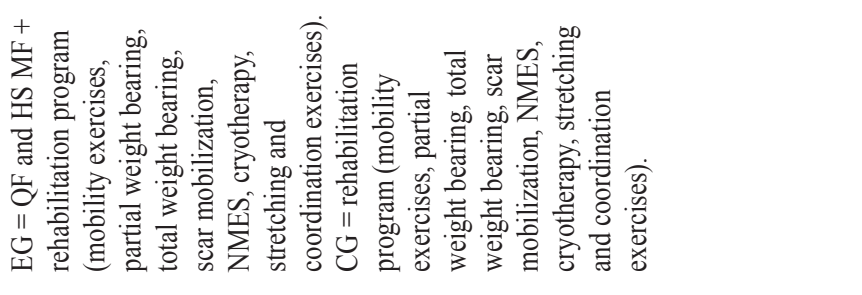 \\
\hline 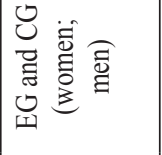 & 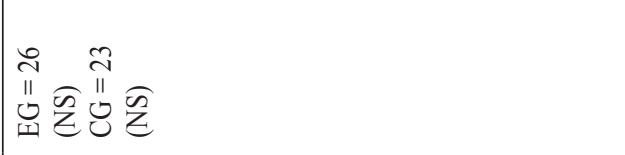 & 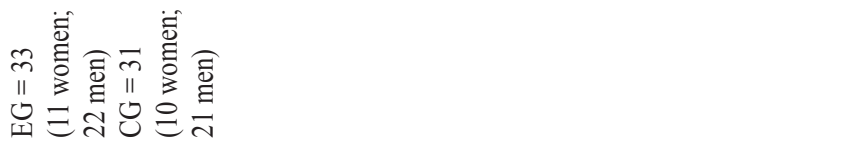 \\
\hline 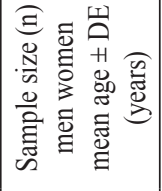 & 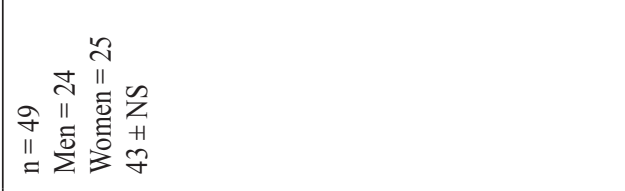 & 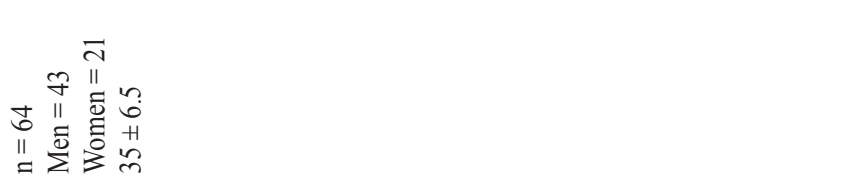 \\
\hline 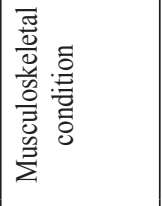 & $\frac{n}{\infty}$ & 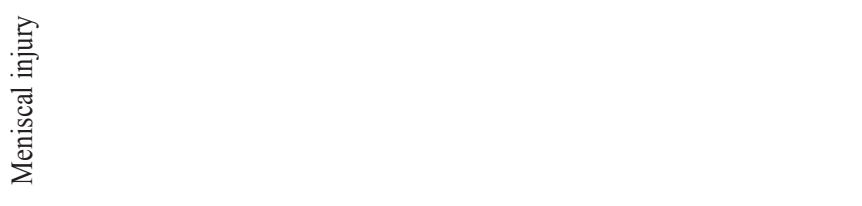 \\
\hline 害䓌言 & 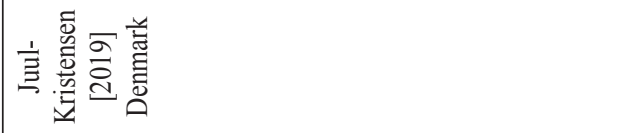 & 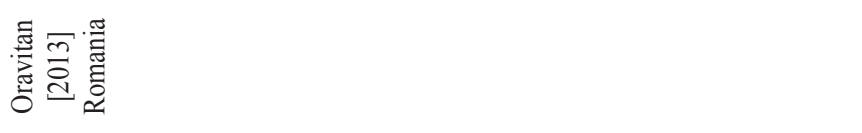 \\
\hline 总 & 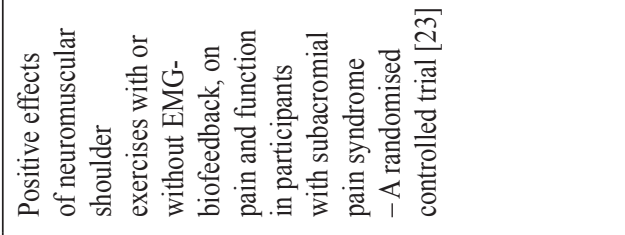 & 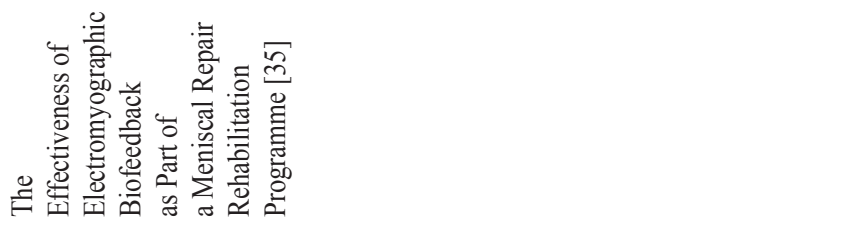 \\
\hline 㞼䓃总 & & \\
\hline
\end{tabular}




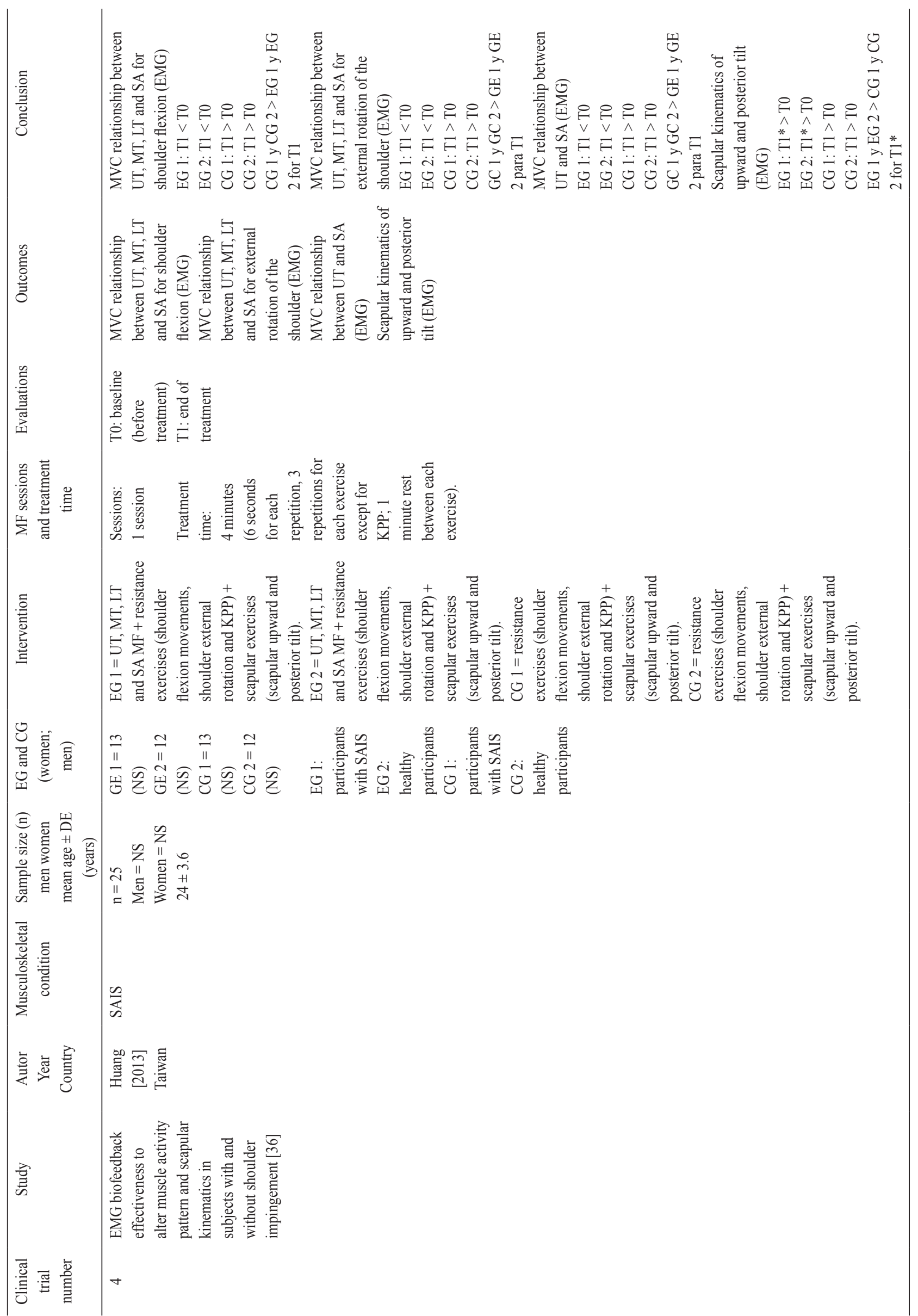




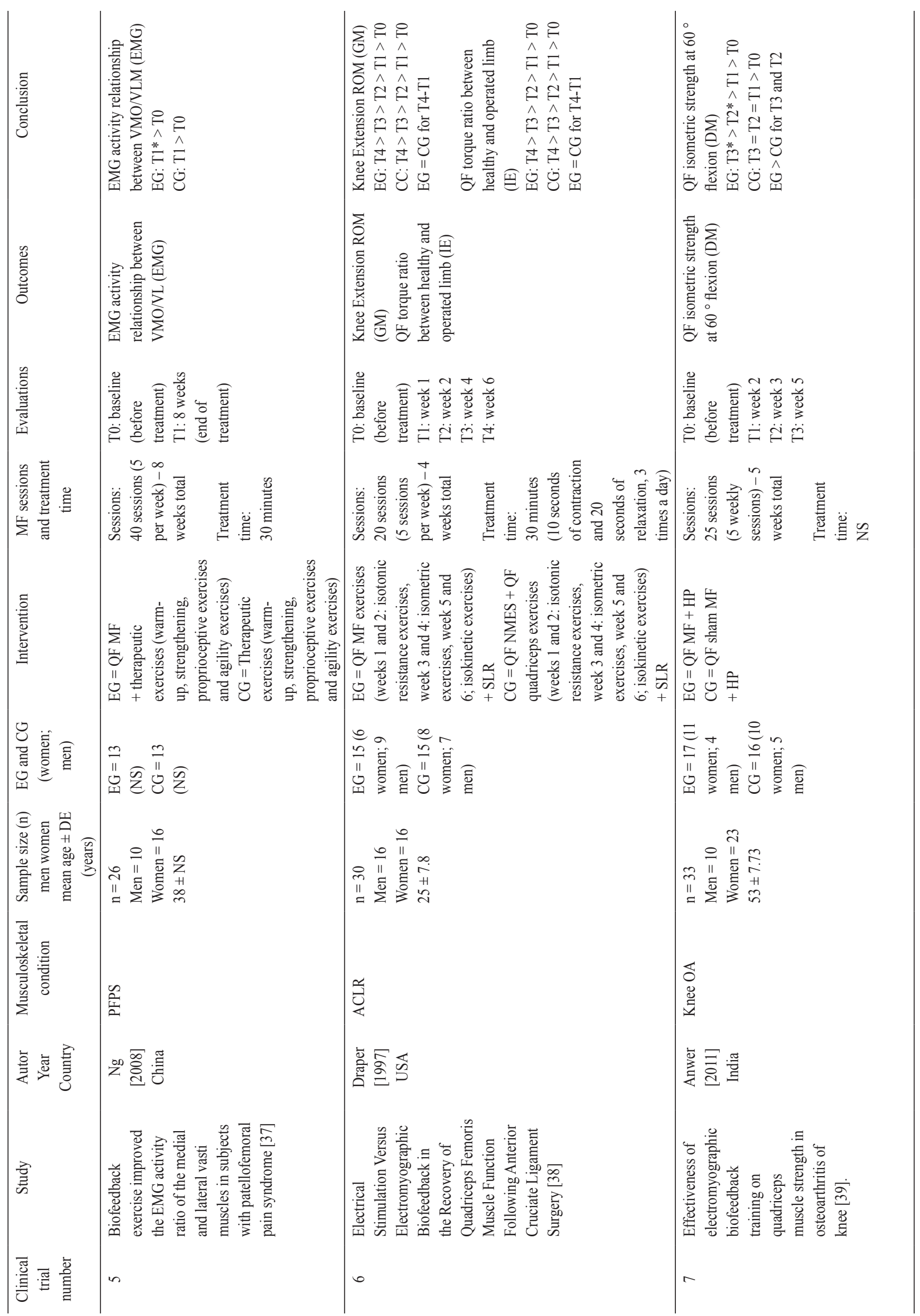




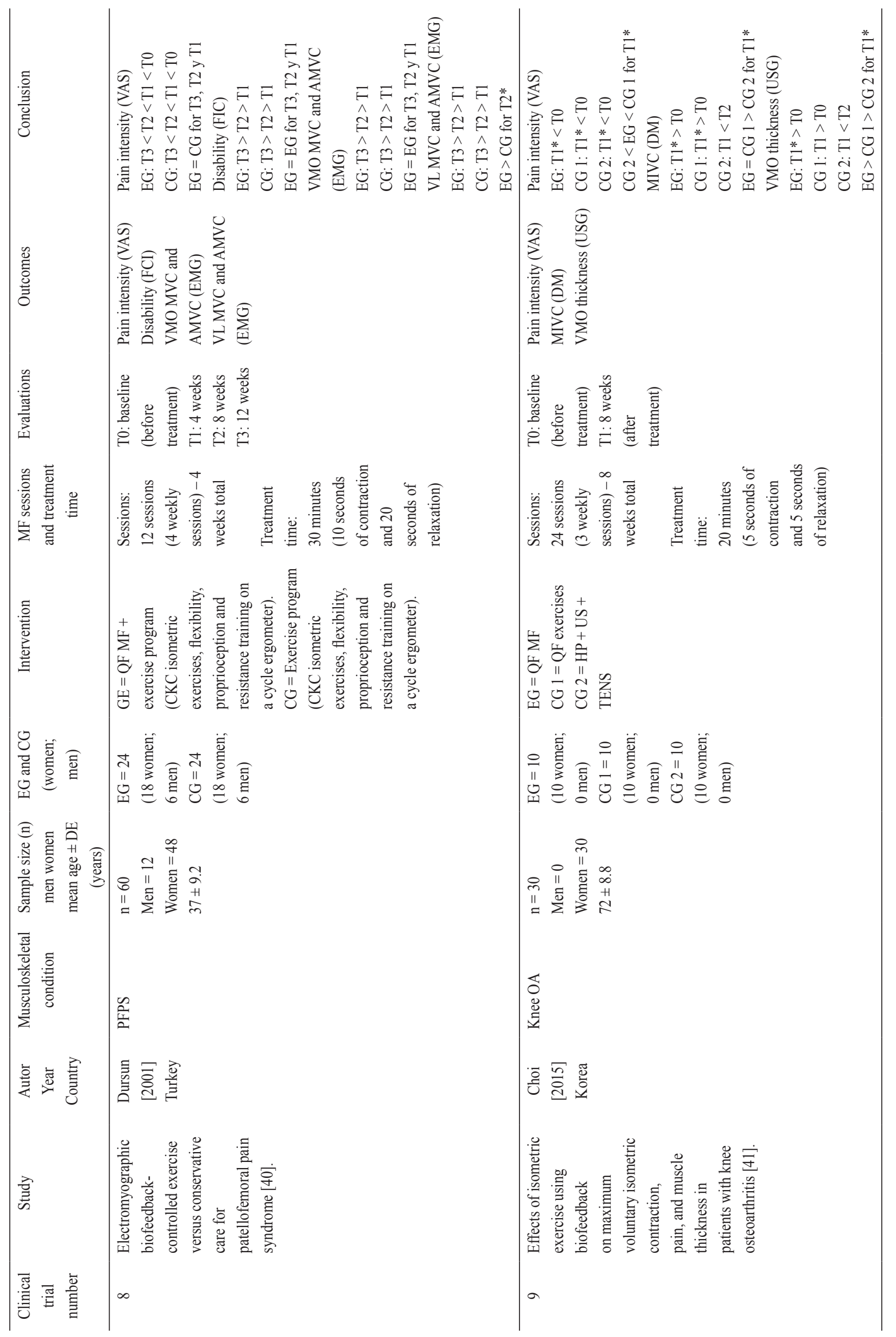




\begin{tabular}{|c|c|c|}
\hline & 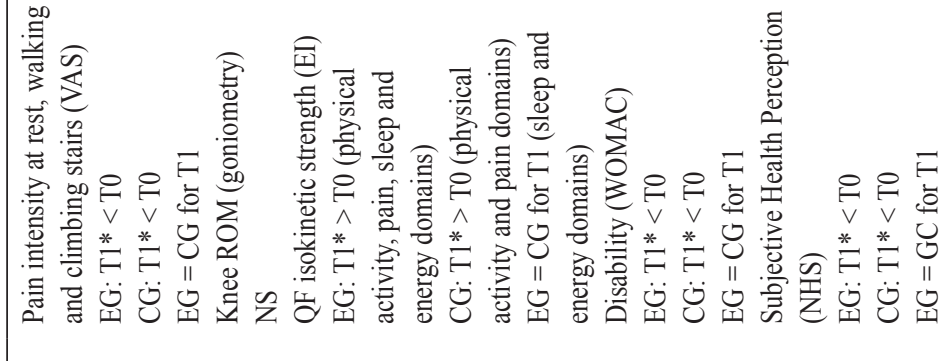 & 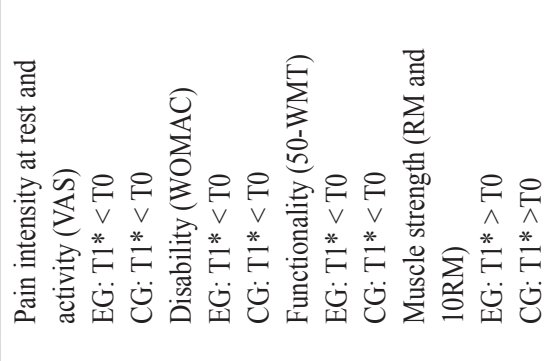 \\
\hline & 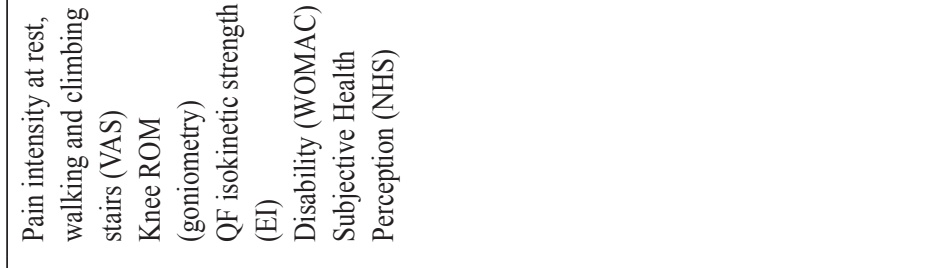 & 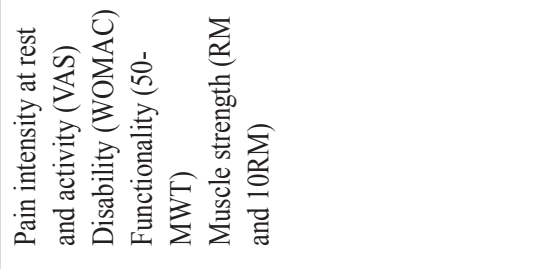 \\
\hline 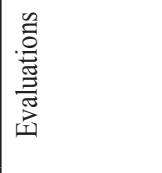 & 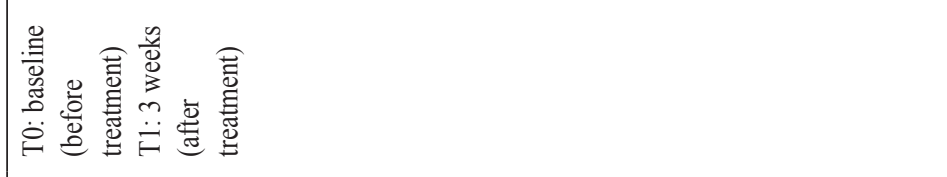 & 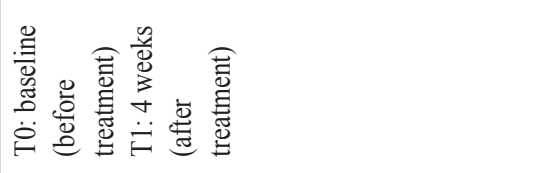 \\
\hline 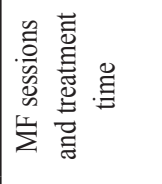 & 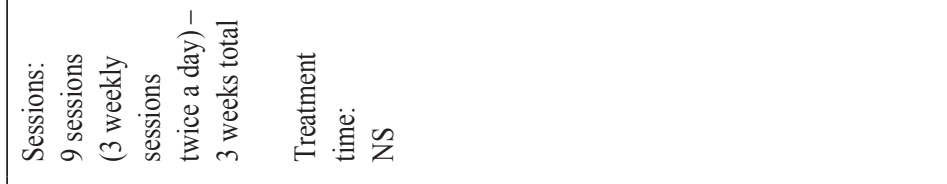 & 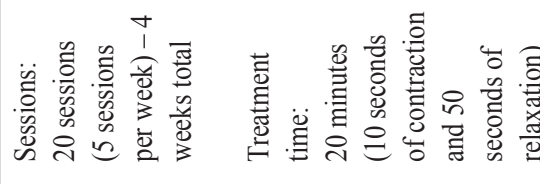 \\
\hline & 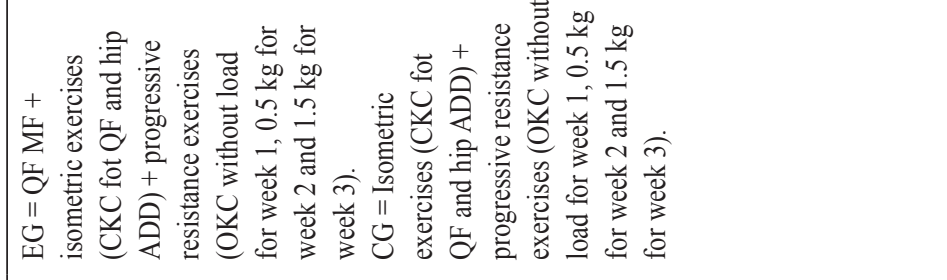 & 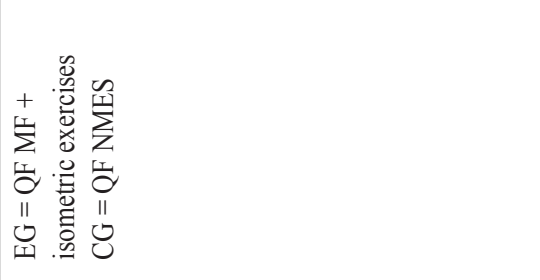 \\
\hline 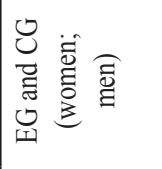 & 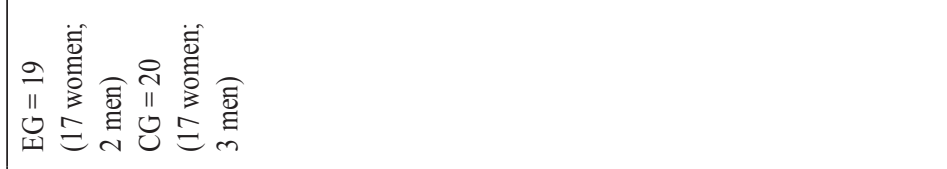 & 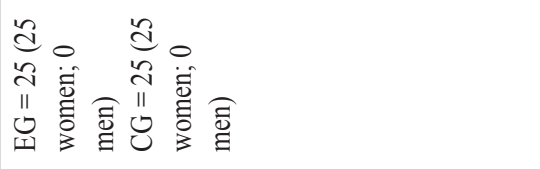 \\
\hline 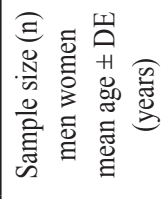 & 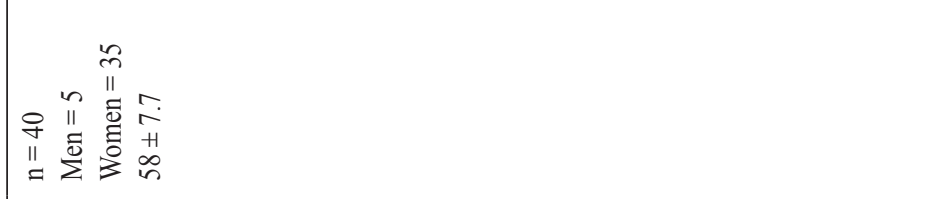 & 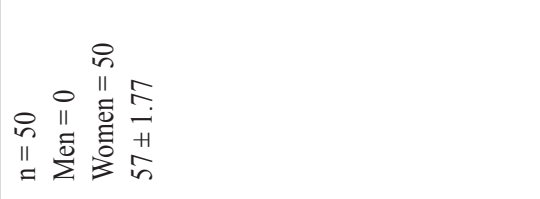 \\
\hline 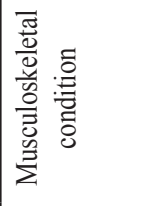 & 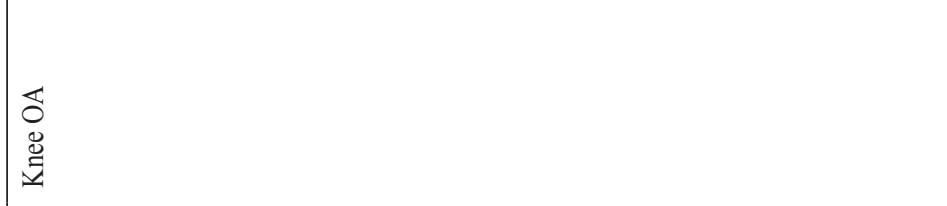 & 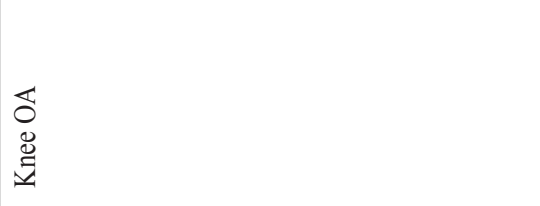 \\
\hline 害总言 & 总完总 & 怠灾总 \\
\hline 悹 & 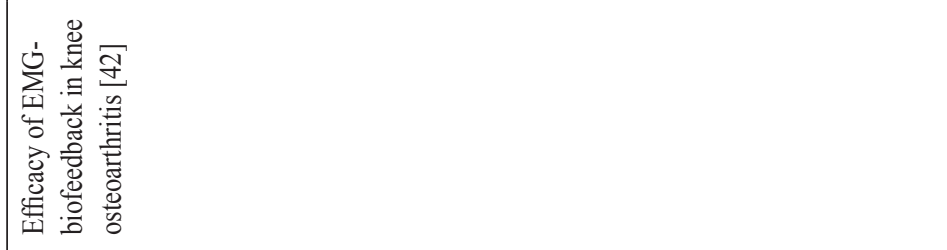 & 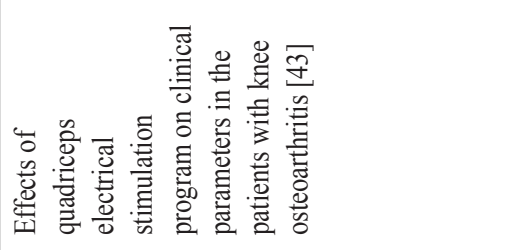 \\
\hline 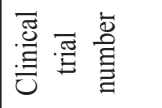 & $\odot$ & \\
\hline
\end{tabular}




\begin{tabular}{|c|c|c|}
\hline & 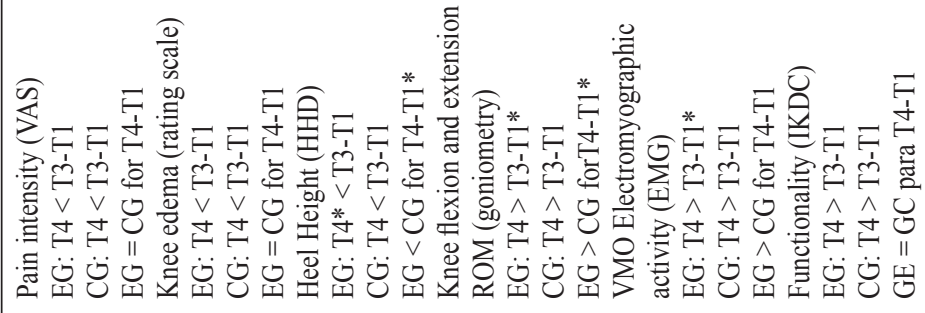 & 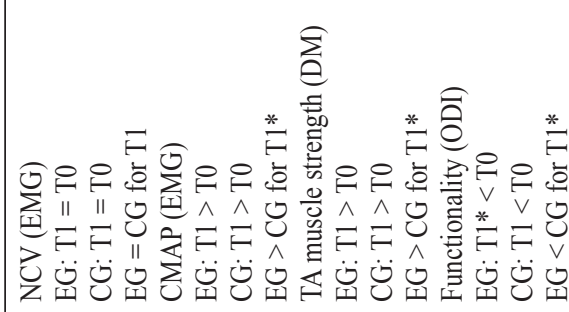 \\
\hline & 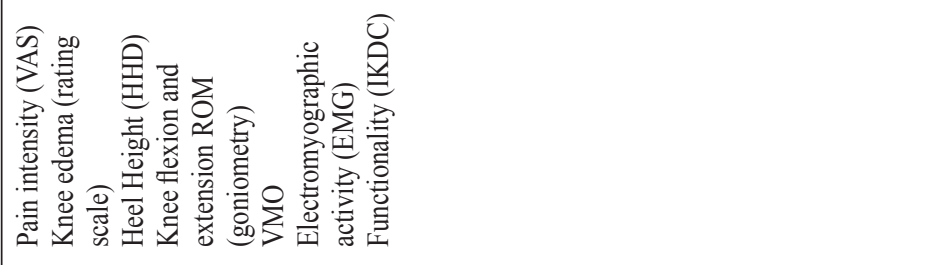 & 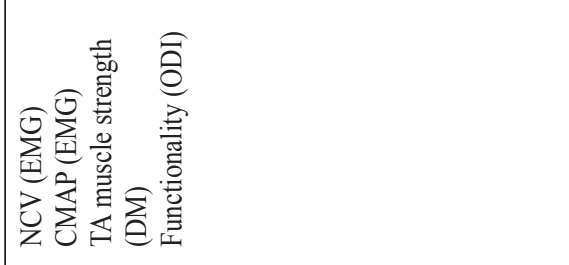 \\
\hline & 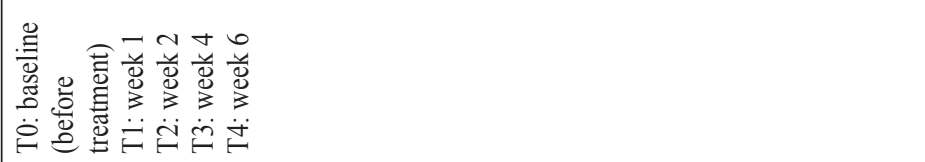 & 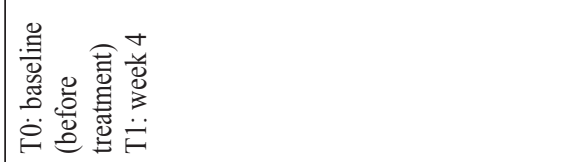 \\
\hline 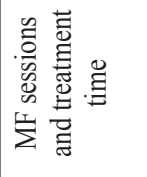 & 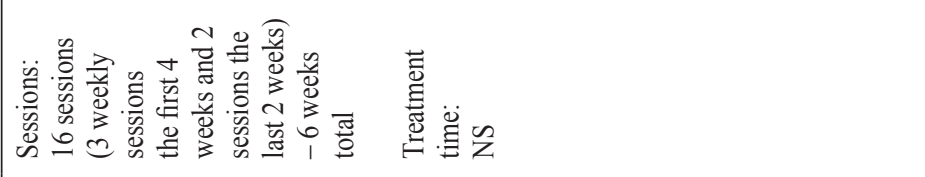 & 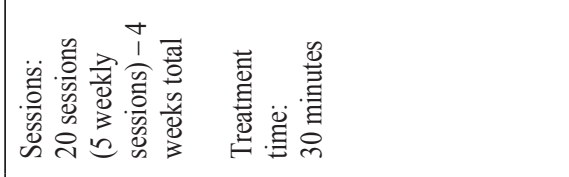 \\
\hline & 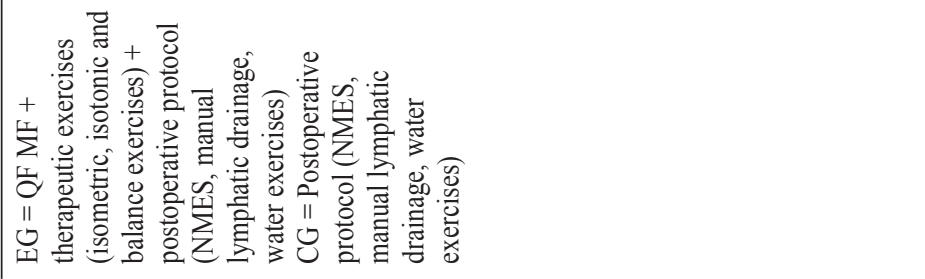 & 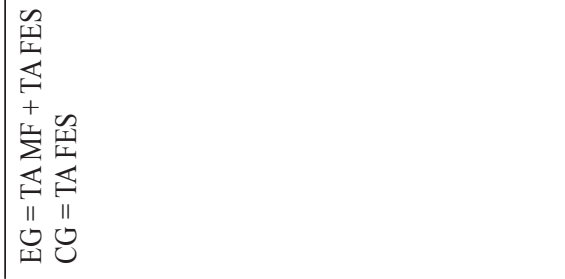 \\
\hline 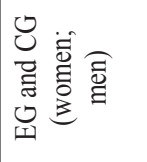 & 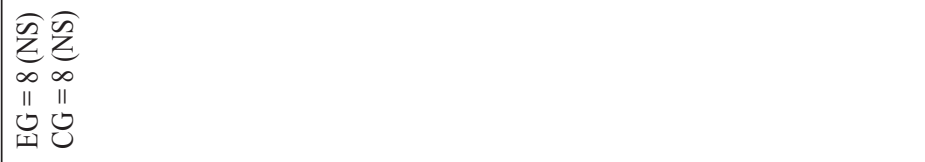 & 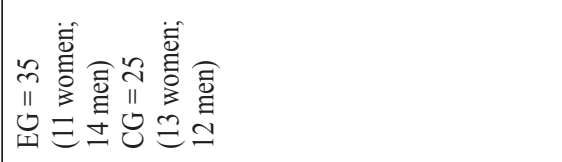 \\
\hline 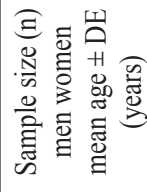 & 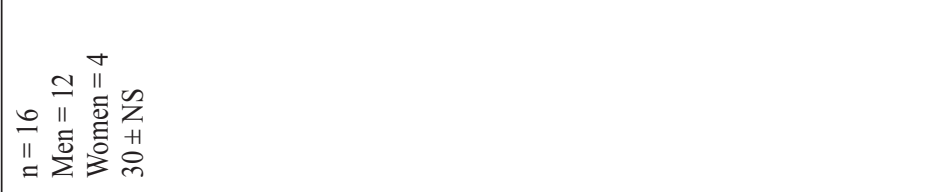 & 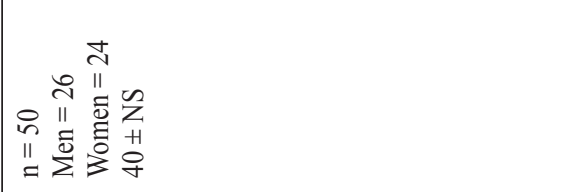 \\
\hline 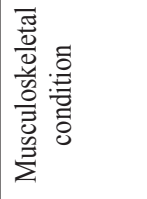 & 范 & 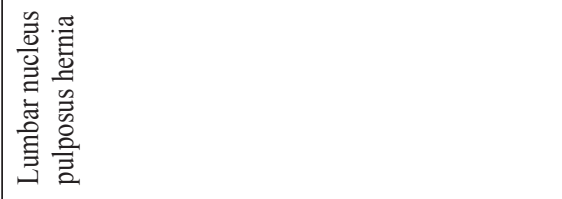 \\
\hline 总䲶言 & 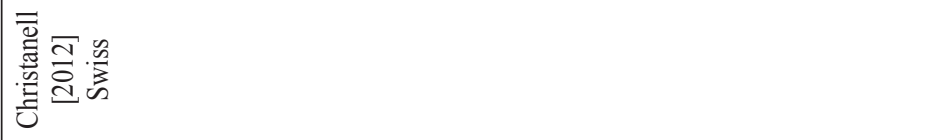 & 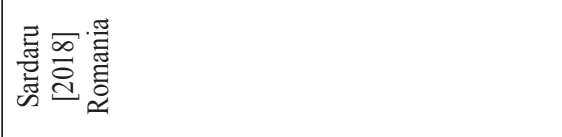 \\
\hline 蛋 & 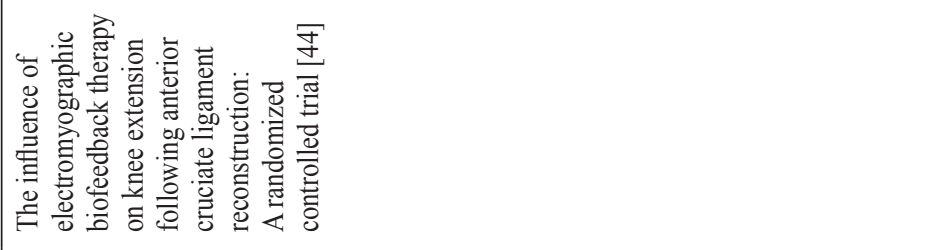 & 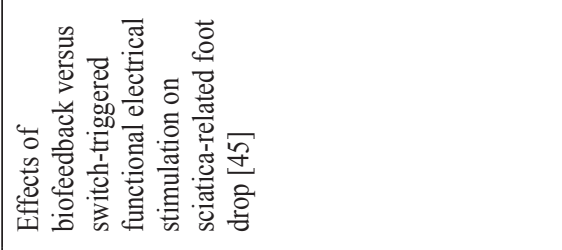 \\
\hline 苞焉离 & $\simeq$ & $\cong$ \\
\hline
\end{tabular}




\begin{tabular}{|c|c|c|}
\hline & 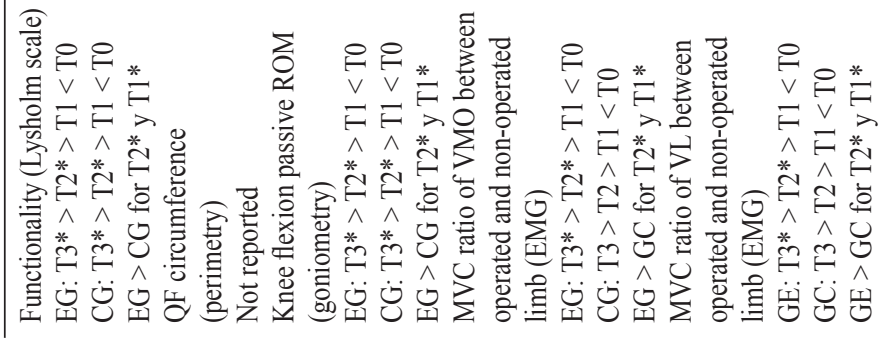 & 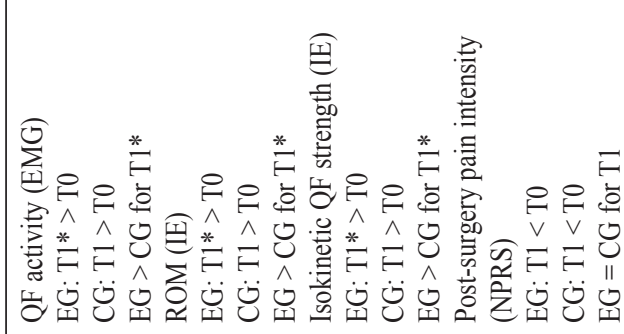 \\
\hline & 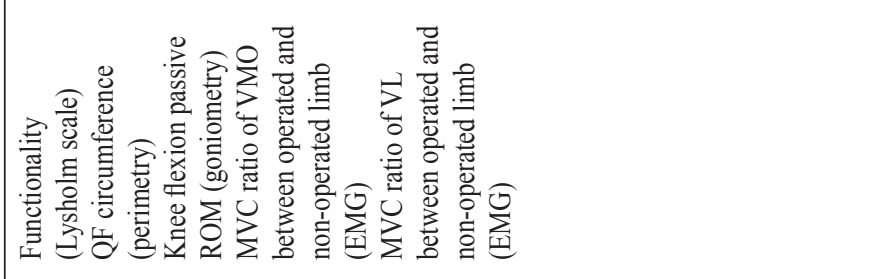 & 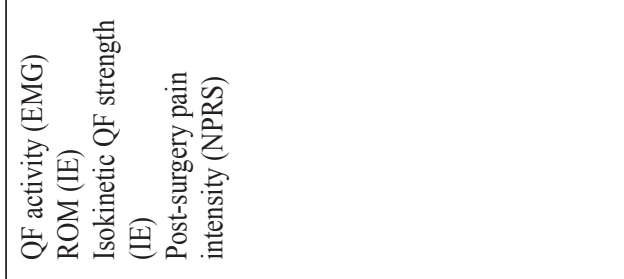 \\
\hline & 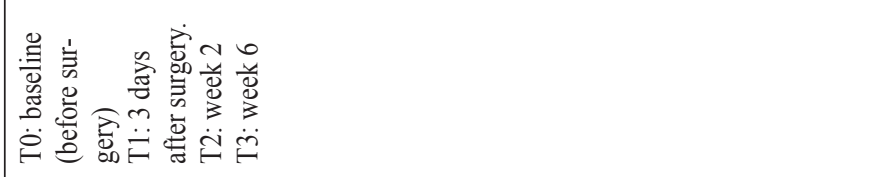 & 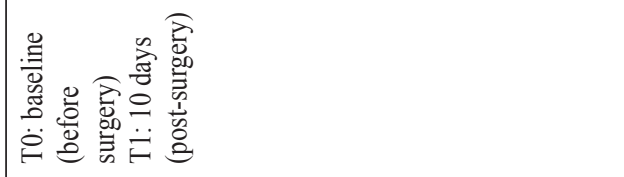 \\
\hline 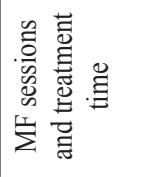 & 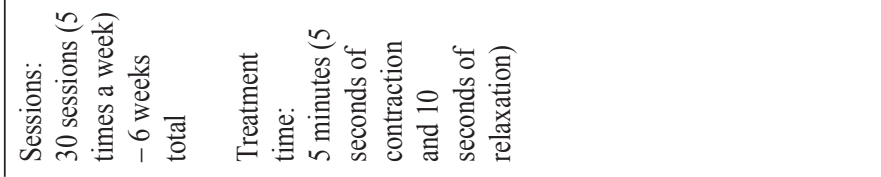 & 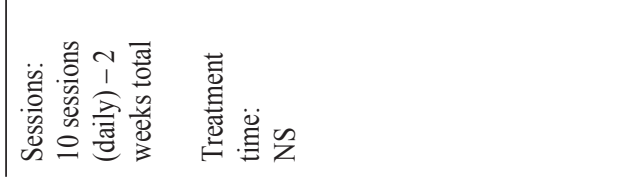 \\
\hline & 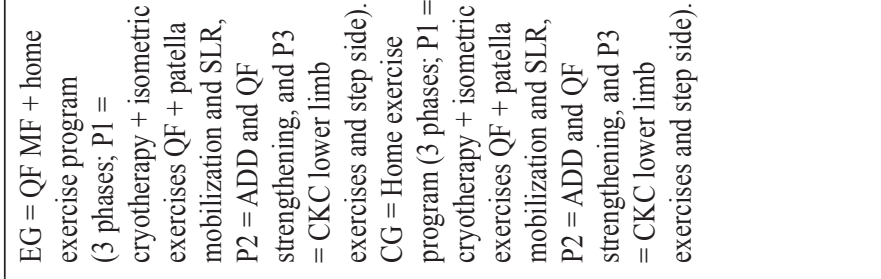 & 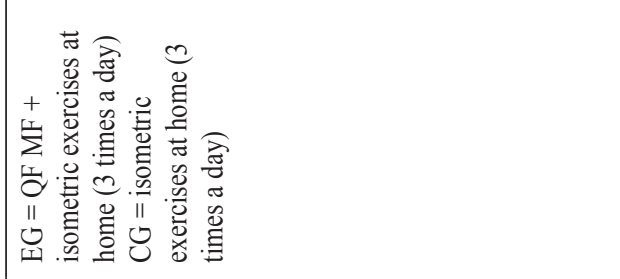 \\
\hline 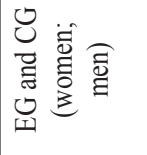 & 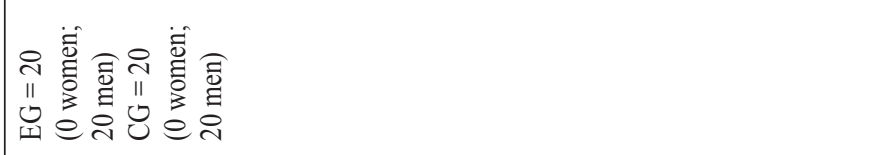 & 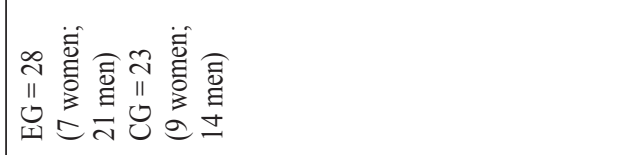 \\
\hline 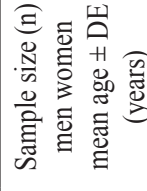 & 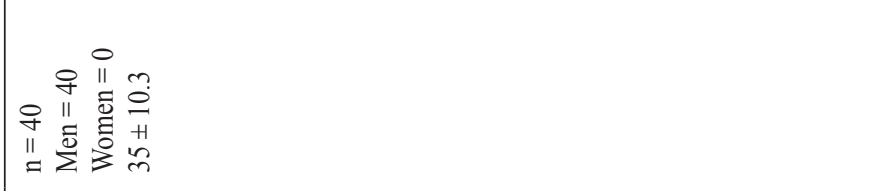 & 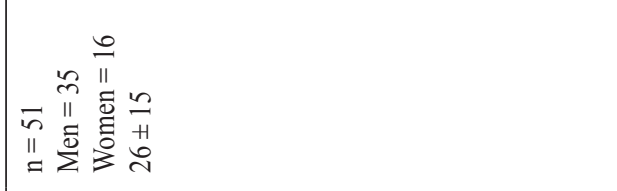 \\
\hline 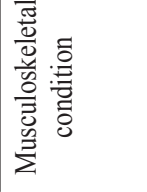 & 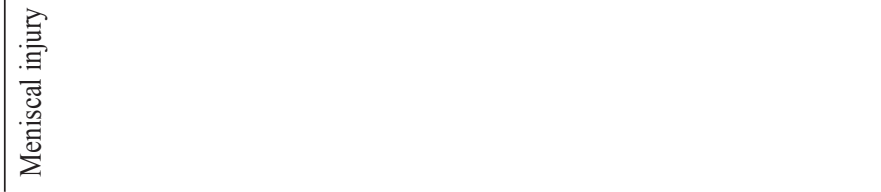 & 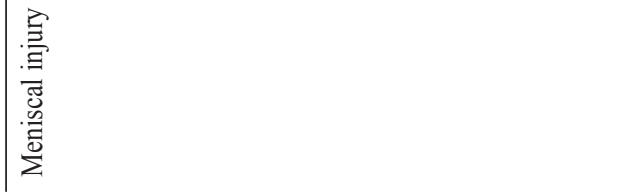 \\
\hline 总鴶言 & 番总总 & 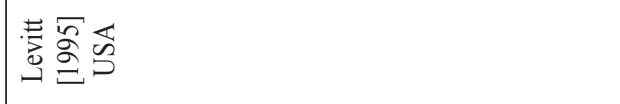 \\
\hline $\begin{array}{l}\text { 胥 } \\
\text { 离 }\end{array}$ & 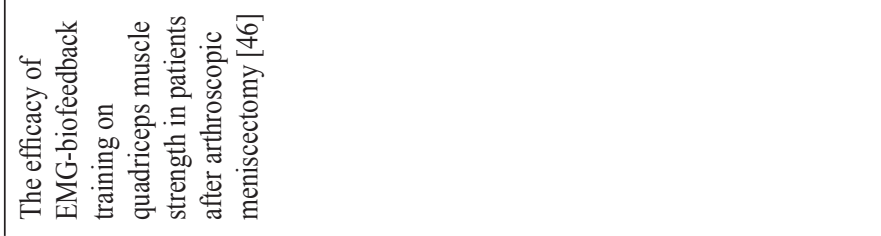 & 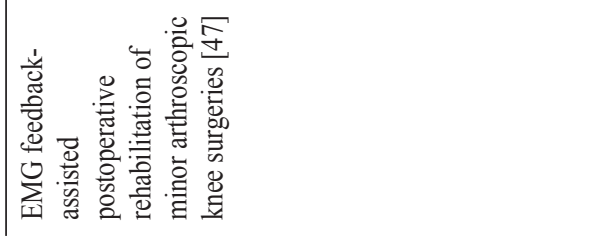 \\
\hline 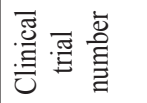 & \pm & \\
\hline
\end{tabular}




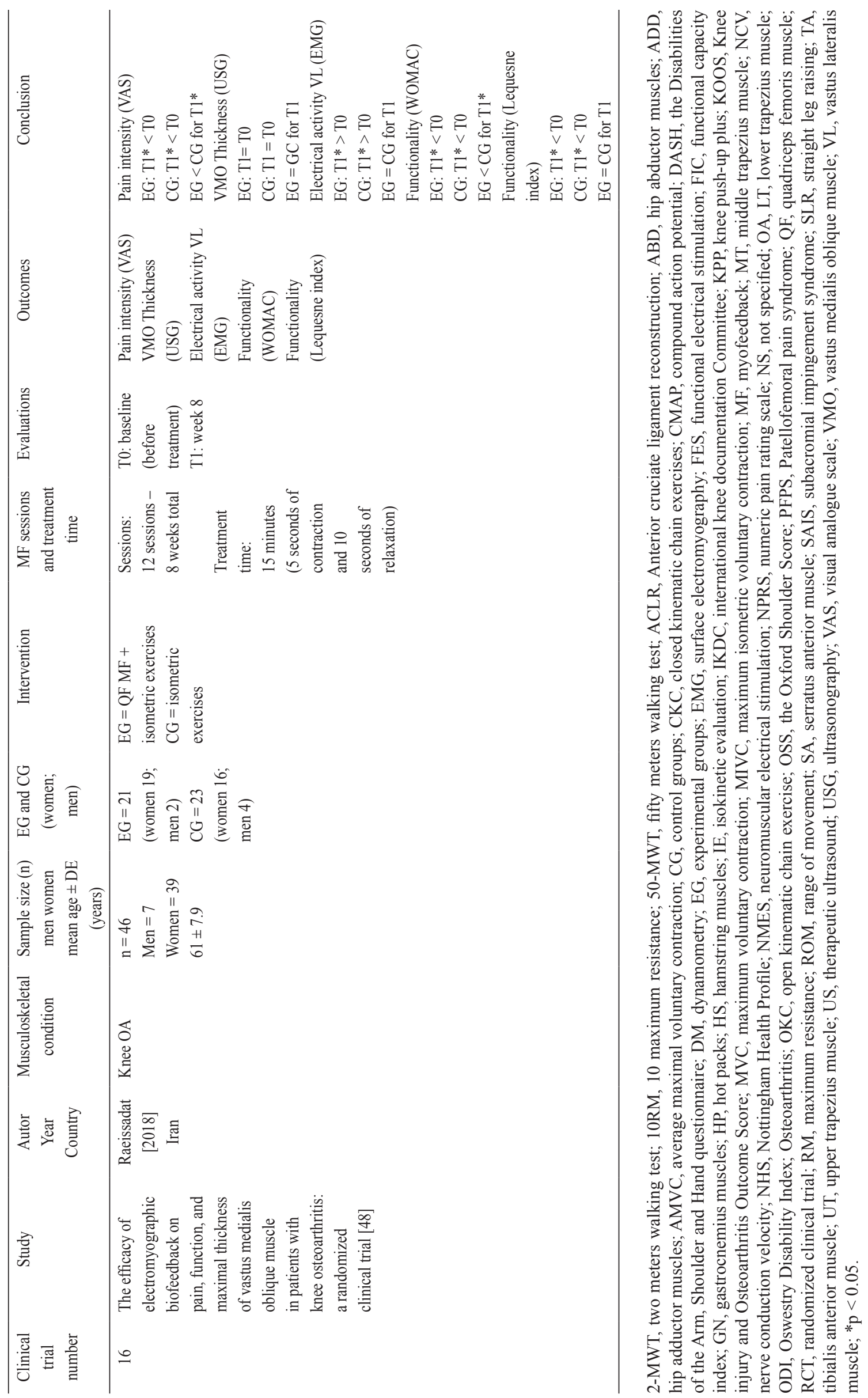




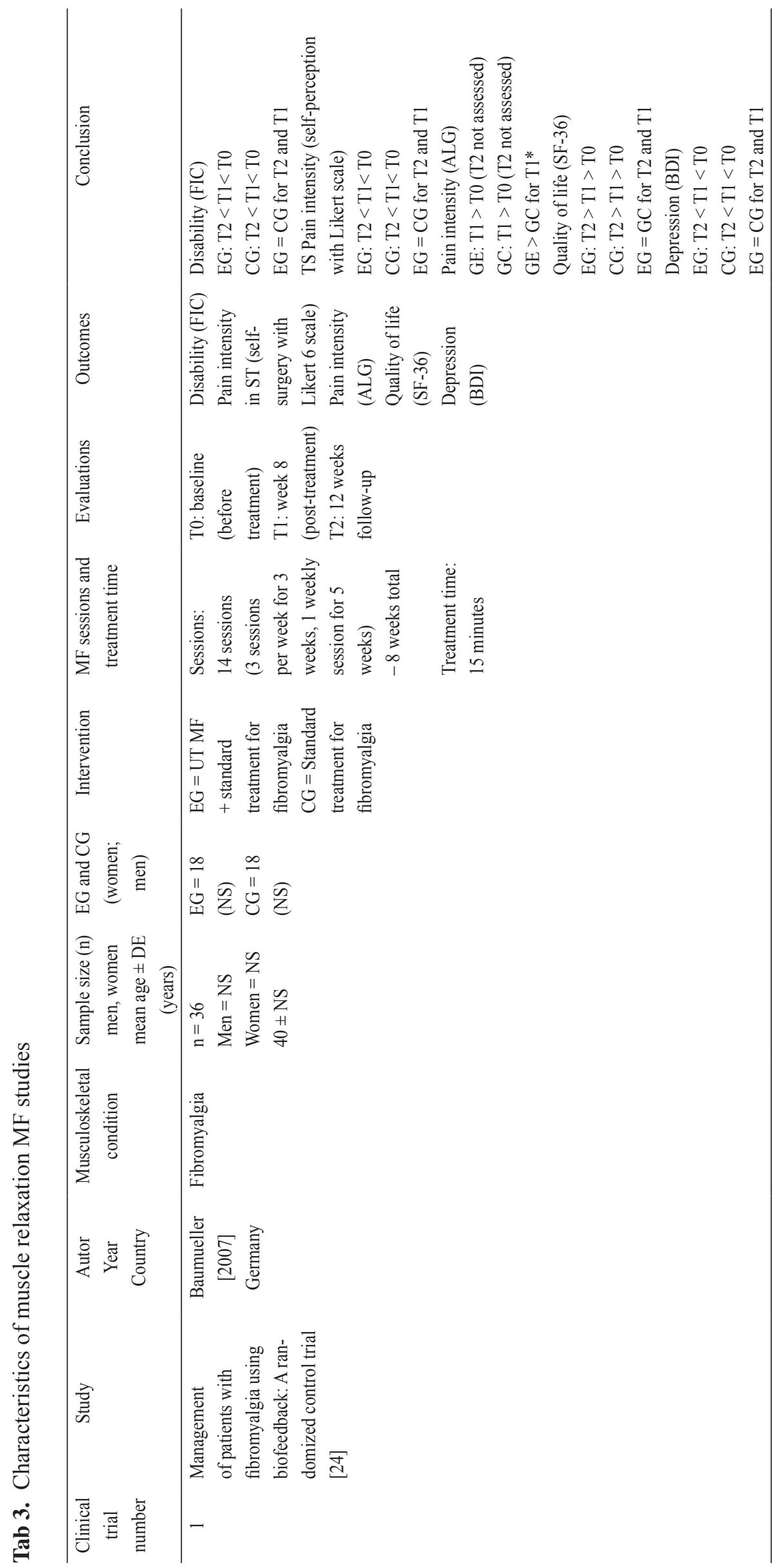




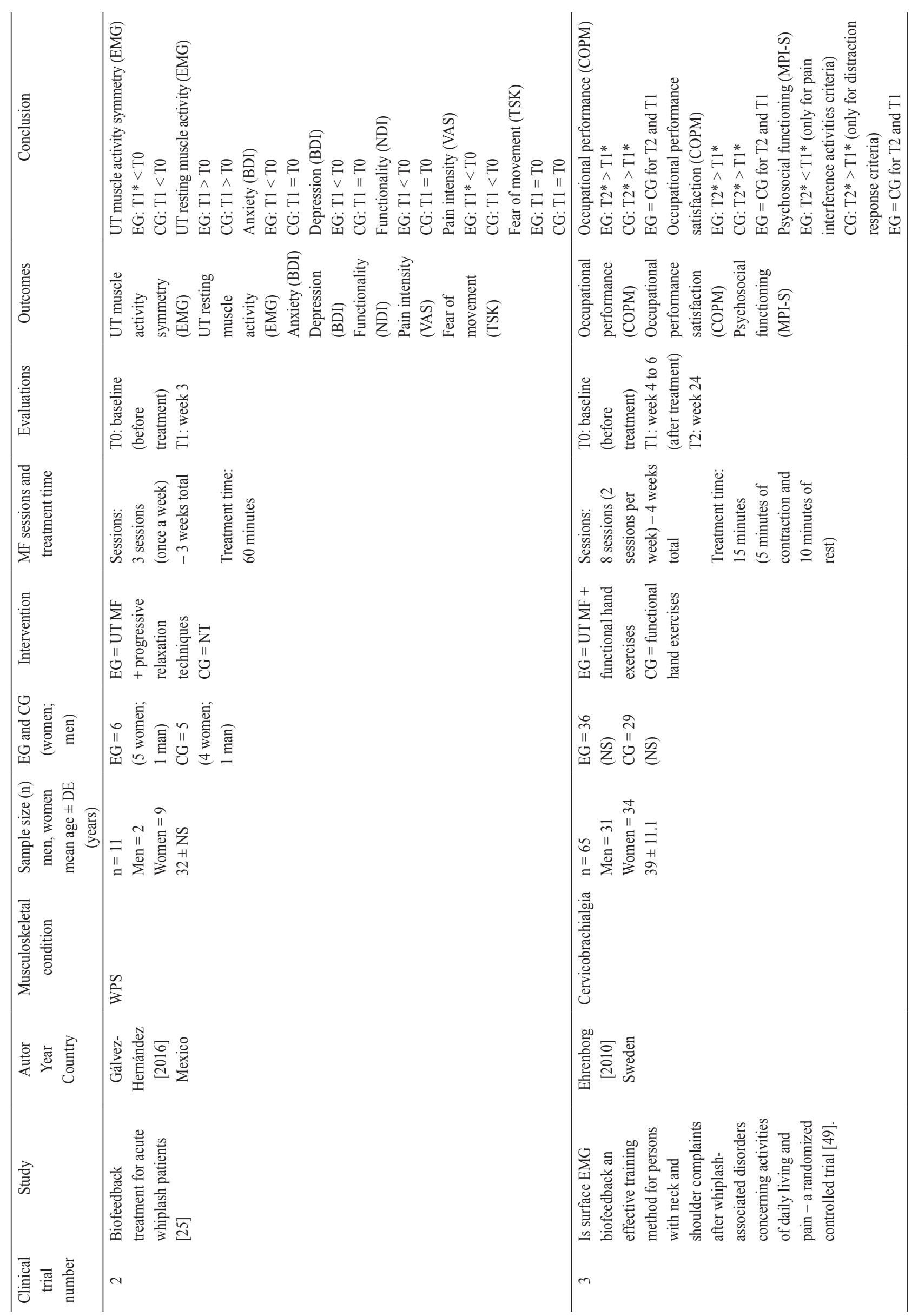




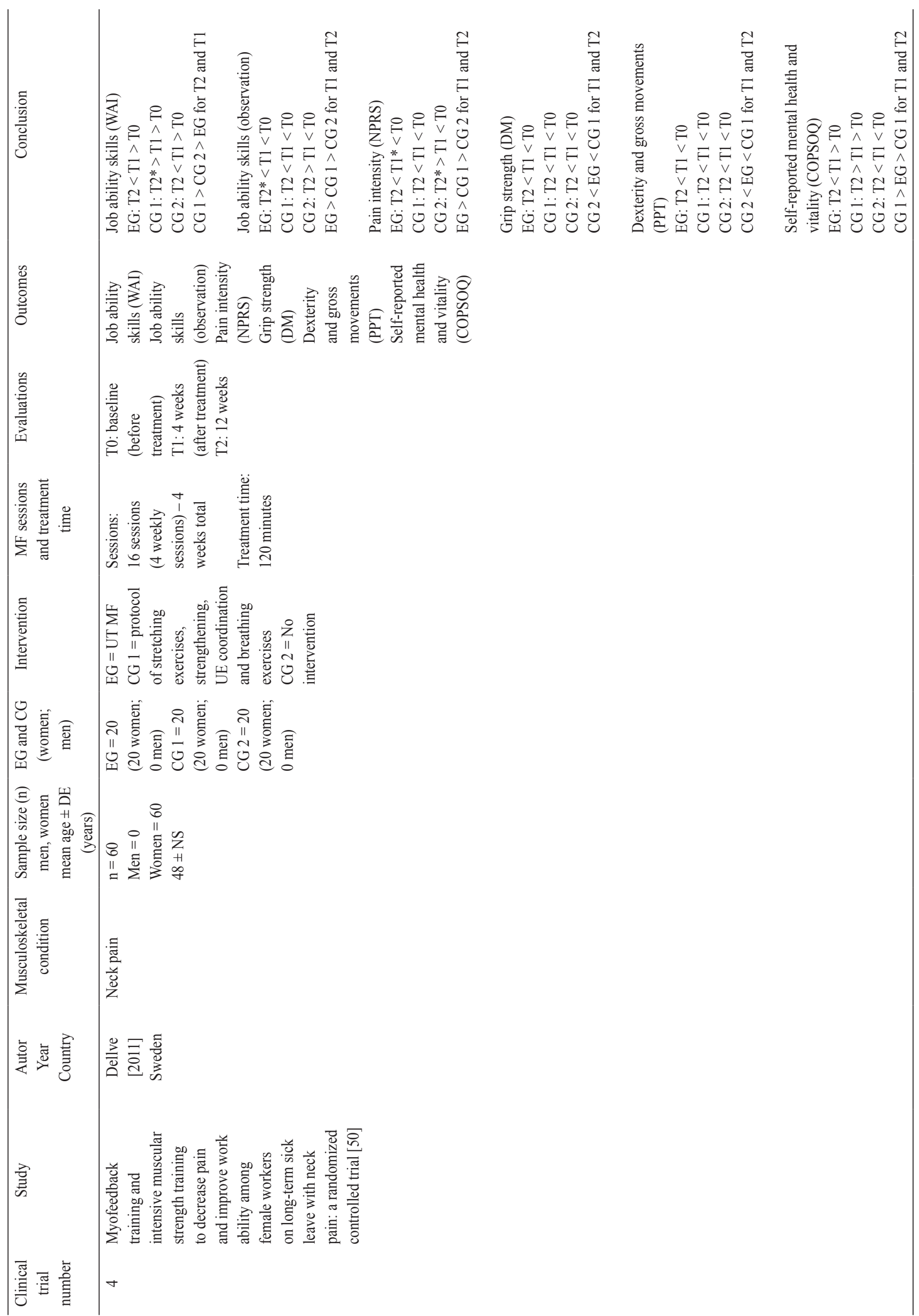




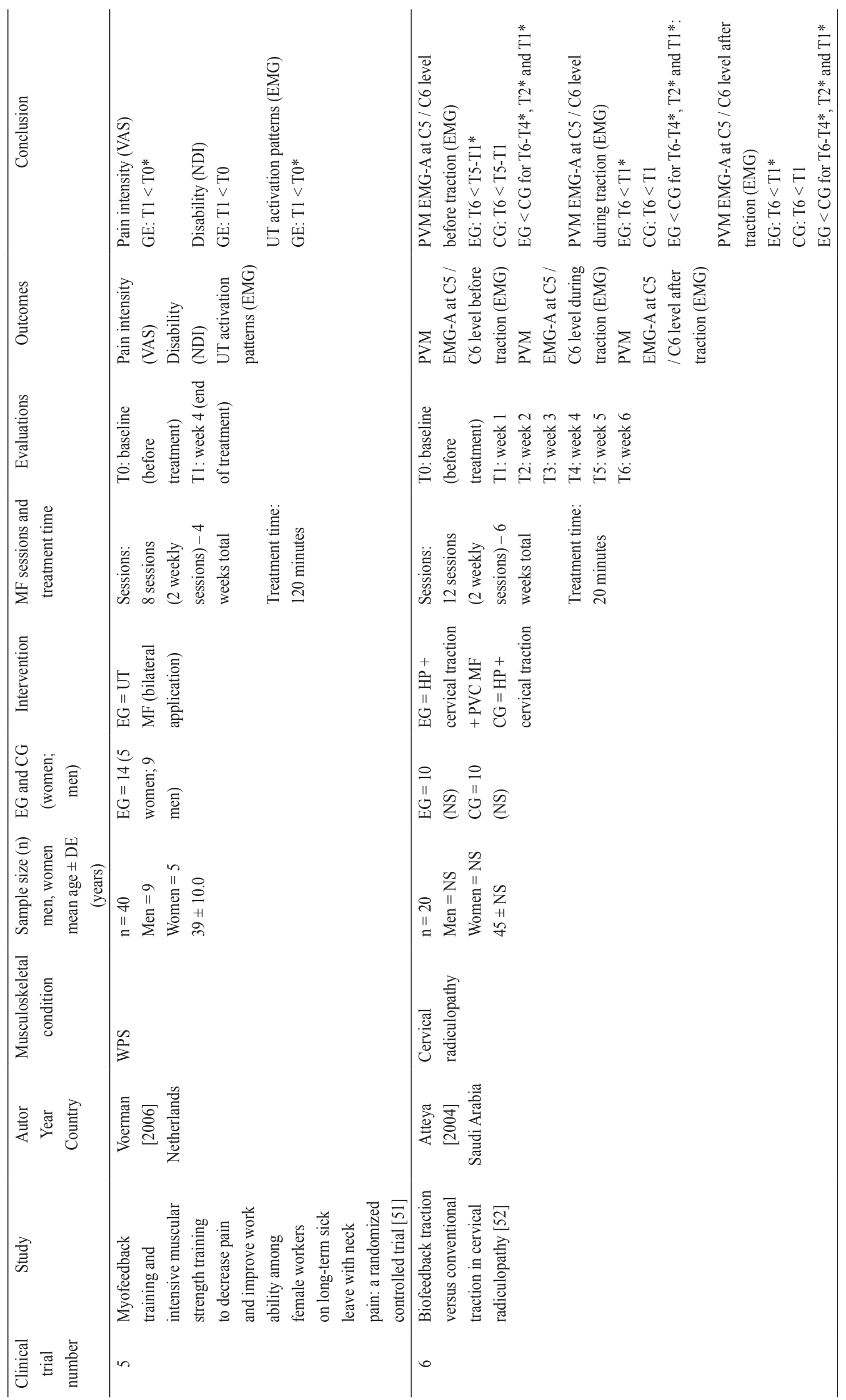




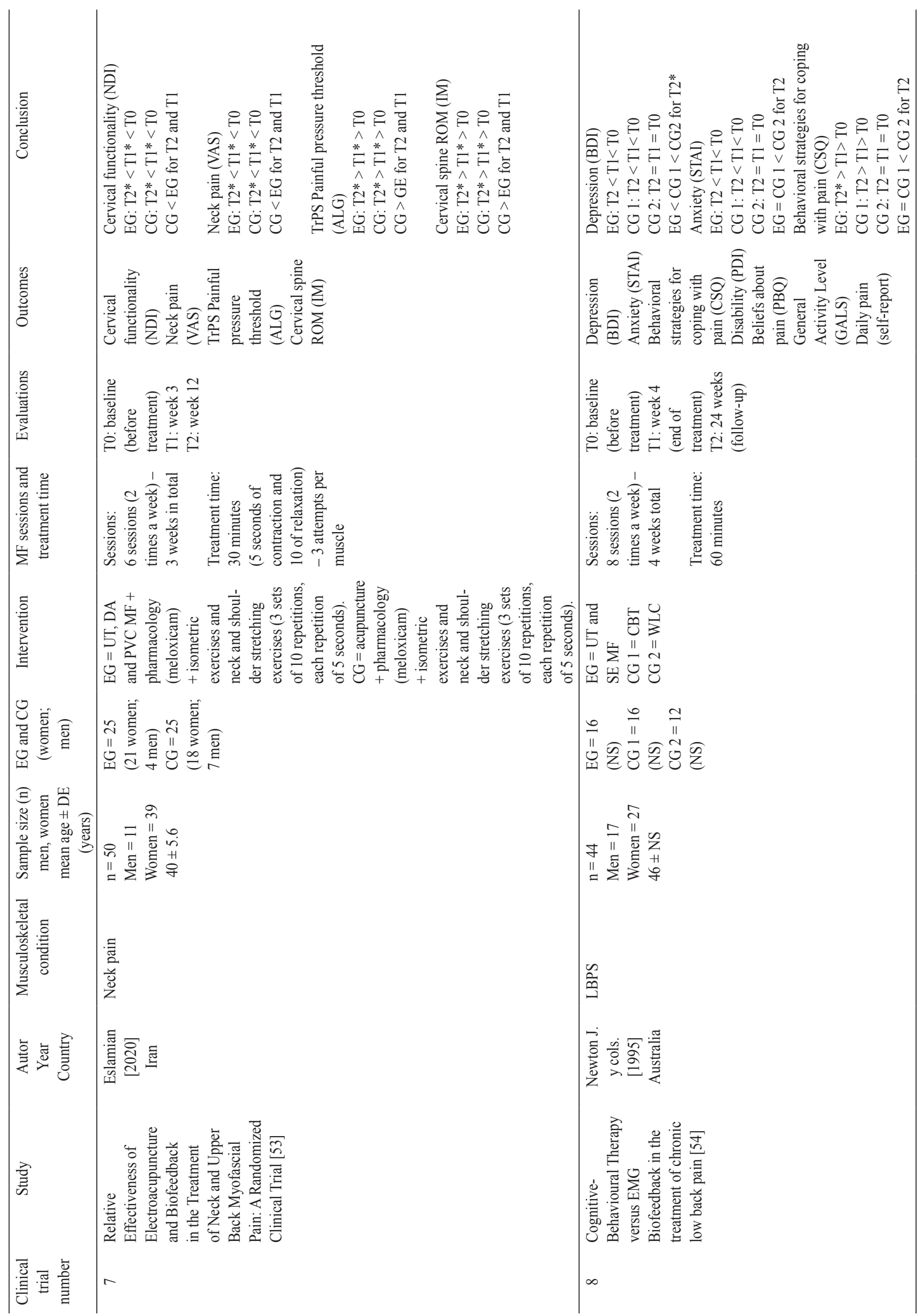




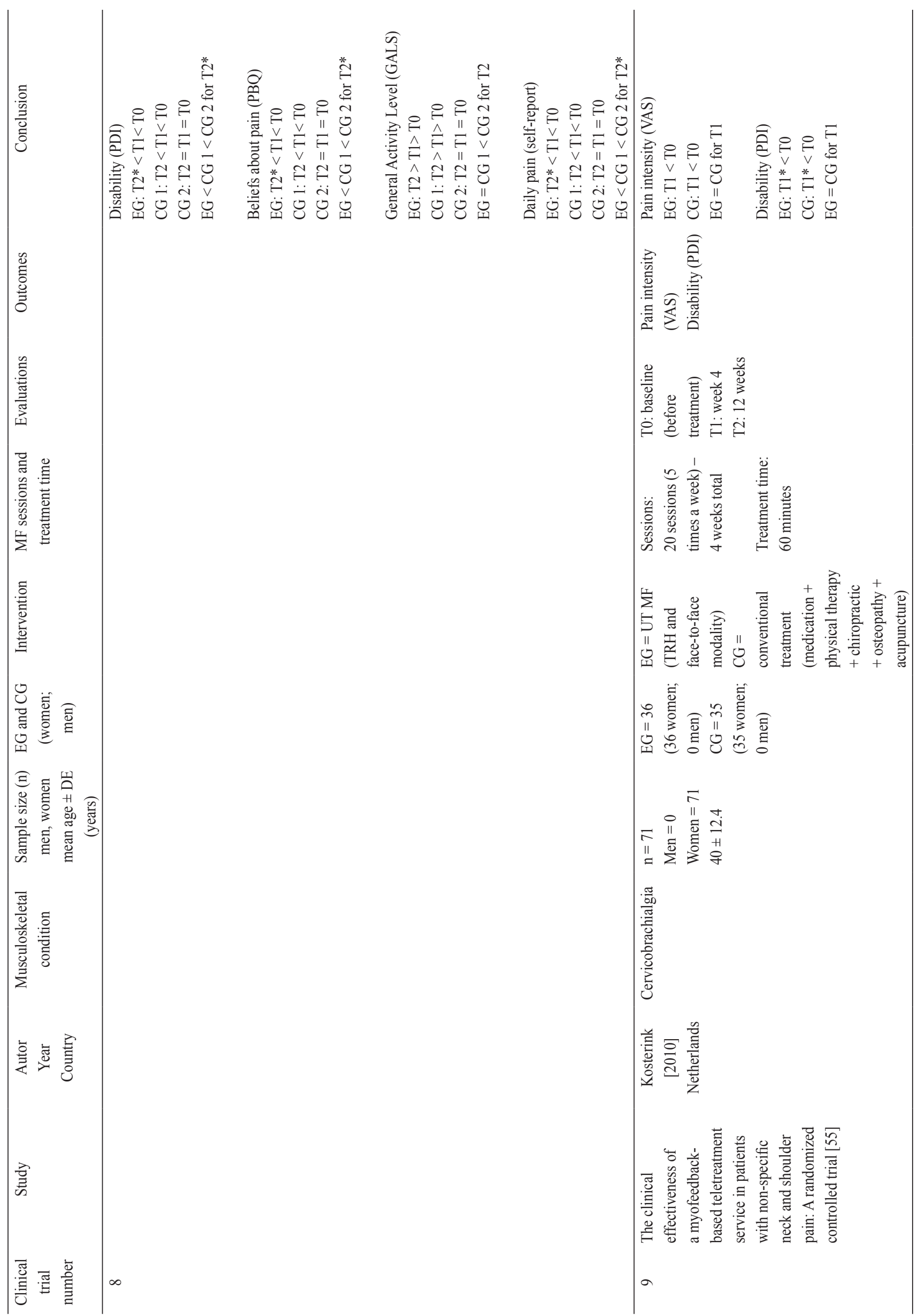




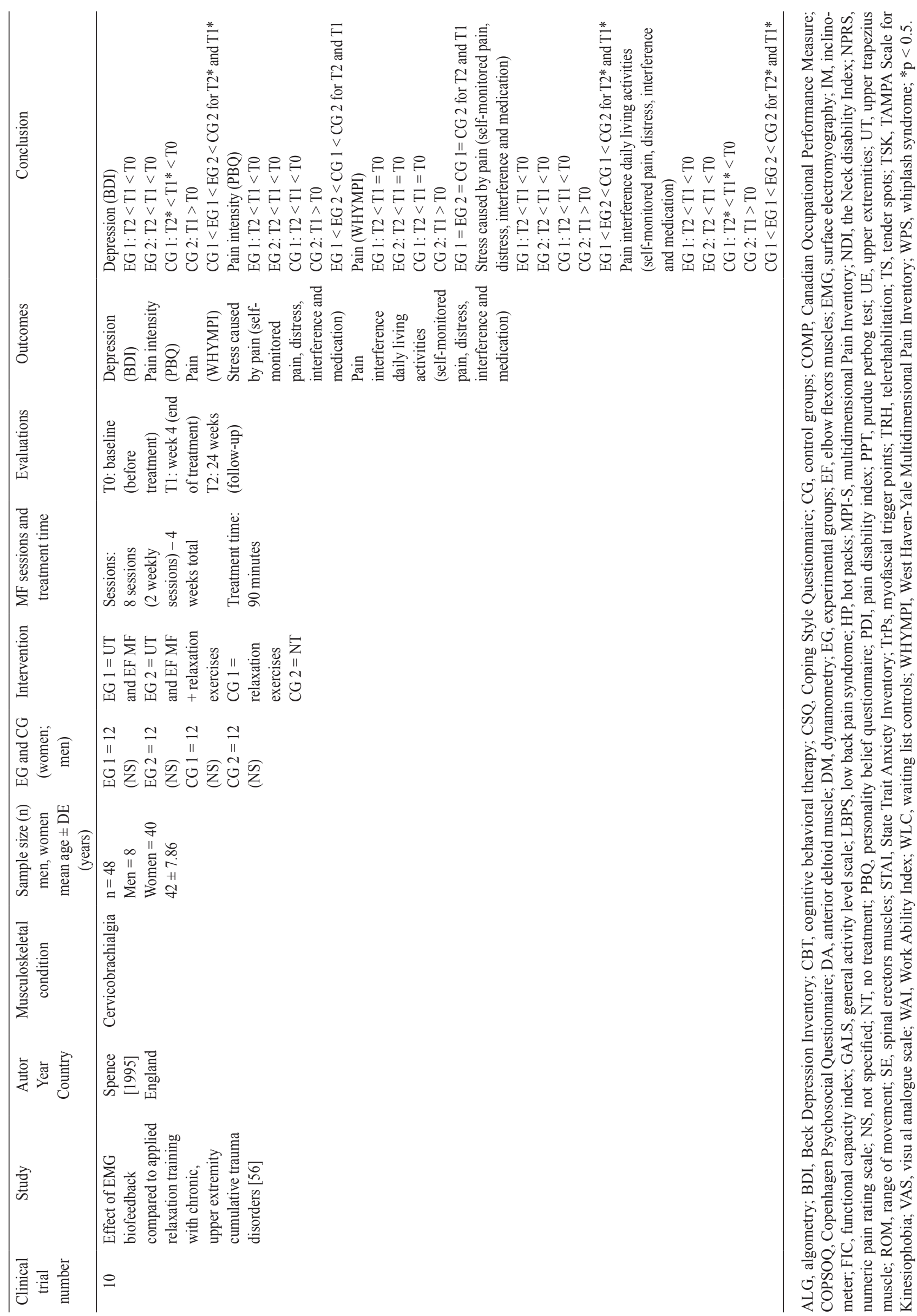


Complementary treatments for muscle strengthening MF included therapeutic exercises (stretching exercises, resistance, mobility, coordination, proprioception or water exercises) $(\mathrm{n}=14,53.84 \%)[22,23,35-37$, 39,40,42-44,46-48] functional electrical stimulation (FES) $(\mathrm{n}=1,6.25 \%)$ [45], neuromuscular electrical stimulation (NMES) $(\mathrm{n}=2,12.5 \%]$ [35,44], lymphatic drainage $(\mathrm{n}=2,12.5 \%)[22,44]$, hot packs $(\mathrm{HP})(\mathrm{n}=1$, $6.25 \%$ ) [28] and cryotherapy $(\mathrm{n}=1,6.25 \%$ ) [46]. On the other hand, complementary treatments for muscle relaxation MF included cervical distraction $(\mathrm{n}=1$, $6.25 \%)$ [41], HP $(\mathrm{n}=1,6.25 \%)$ [41], relaxation techniques $(\mathrm{n}=1,6.25 \%)$ [56] and therapeutic exercises (stretching and functional exercises) $(\mathrm{n}=2,12.5 \%)$ $[49,53]$. Only Eslamian (2020) used NSAIDs in participants with neck pain in addition to MF relaxation [53].

The control groups in the strengthening MF studies received therapeutic exercises (resistance exercises, flexibility, functional and home exercises) [22,23,35-38,40,42,46-48], NMES [22,38,43,44], FES [45], superficial thermotherapy (HP) [50,52], TENS [41], US [41], postoperative lymphatic drainage and water exercises [44]. In addition, it should be noted that no articles reported the use of drugs for CG. On the other hand, for CG in muscle relaxation MF studies, superficial thermotherapy (HP) [52], joint distraction [52], acupuncture [53], therapeutic exercises (isometric exercises, stretching and relaxation) $[49,53,56]$, cognitive behavioral therapy (CBT) [54], joint manipulation [55], and medication $[53,55]$ were used. The studies by Dellve (2011), Spence (2016) and Gálvez-Hernández (1995) did not administer treatment for CG $[25,50,56]$.

Regarding the treatment sessions, an average of 5 sessions was observed for strengthening MF and 8 sessions for relaxation MF. Minimum sessions for strengthening MF studies were 1 (Huang, 2013) [36] with a maximum of 30 (Kirnap, 2005) [46], while minimum sessions for muscle relaxation MF studies were 3 (Gálvez-Hernández, 2016) [25] with a maximum of 16 (Dellve, 2011) [50]. Strengthening studies $(\mathrm{n}=9$, $56.25 \%$ ) sessions were mostly carried out in continuous days in an average of 5 weeks $[23,24,26,27,28,32$, 34-36], while muscle relaxation MF studies $(n=9$, $90.00 \%$ ) included interval sessions averaging 5 weeks [24,25,50-56].

The average treatment time for strengthening MF was 20 to 30 minutes, with a minimum of 4 minutes reported by Huang [36] and a maximum of 30 minutes $[37,38,40,45]$, while the average treatment time for relaxation MF was between 40 and 50 minutes, with a minimum of 5 minutes reported by Ehrenborg (2010) [51], and a maximum of 120 minutes by Dellve [50].

The review revealed, for most of relaxation MF studies, 3 evaluation sessions (before and two after treatment; T0, T1 and T2) $(\mathrm{n}=8,80.00 \%)$ [50-56], while the strengthening studies documented mostly 2 evaluation sessions (before and after treatment; T0 and T1) $(\mathrm{n}=10,62.50 \%)[23,35-37,30-43,45,47,48]$. On the other hand, Draper (1997) and Christanell (2012) carried out 5 evaluation sessions for strengthening MF (T0-T4) [38,44], while Atteya (2004) reported 7 sessions for relaxation MF (T0-T6) [52]. An average evaluation time of 1 week between sessions is highlighted for both MF applications.

\section{Main outcomes}

\section{Strengthening MF studies}

The main outcomes of the strengthening MF studies included functionality (disability) $(\mathrm{n}=12,75.0 \%)$ $[22,23,40,42-46,48]$, pain intensity $(\mathrm{n}=9,56.25 \%)$ $[22,23,35,40-44,47,48]$, gait speed $(\mathrm{n}=1,3.8 \%)$ [22], range of motion (ROM) changes $(\mathrm{n}=6,37.5 \%)$ $[22,36,38,42,44,46]$, decreased edema $(n=2,12.5 \%)$ $[22,44]$, changes in electromyographic activity $(n=13$, $81.25 \%$ ) [22,23,35,36,40-42,44-48], muscle strength changes $(\mathrm{n}=7,43.75 \%)[35,38,39,41,43,44,47]$, muscular thickness $(\mathrm{n}=3,18.75 \%)[41,46,48]$ and subjective health assessment $(n=1,0.06 \%)$ [42]. Functionality was assessed with the Lyshom scale $[22,46]$, the Knee injury and Osteoarthritis Outcome Score (KOOS) [46], the hand, arm and shoulder disability index (DASH) [23], the Oxford shoulder scale (OSS) [23], functional capacity index (FCI) [40], 50-meter walk test (50WMT) [43], the Western Ontario and McMaster questionnaire (WOMAC) $[42,43,48]$, the International Knee Documentation Committee (IKDC) guideline [44], the Oswestry Disability Index (ODI) [45] and Lequesne Index [48]. On the other hand, pain intensity was evaluated with the visual analog scale (VAS) $[22,40$,41-44,48], KOOS [35] and numerical pain rating score (NPRS) $[23,47]$, while gait speed was assessed with the 2-meter walk test (2-WMT) [22]. Electromyographic activity (EMG-A) was evaluated with surface electromyography $[23,35-37,40,44,45,47,48]$, while muscle strength assessment was performed through dynamometry $[35,39,41,45,47]$, isokinetic assessment $[38,42]$ and maximum resistance estimation (Rmax) [43]. Muscle thickness was examined by ultrasonography $[41,48]$ and muscle circumference measurement [46], while perimetry was used to quantify edema $[22,44]$. Subjective health assessment was assessed with the Nottingham Health Profile (NHS) [42].

Results show an improvement in intragroup functionality for Lyshom, KOOS, OSS, DASH, IKDC, ODI, WOMAC, and Lequesne index, although with statistical significance in favor of EG (intergroup) for meniscal rehabilitation studies (Lyshom scale, $\mathrm{p}<0.001$ ) 
$[22,46]$, knee OA $(50-\mathrm{WMT}, \mathrm{p}<0.01$; WOMAC, $\mathrm{p}<0.05)[42,43]$, and lumbar hernia (ODI, $\mathrm{p}<0.0001$ ) [45]. MF applications focused on quadriceps femoris strengthening for knee conditions, and tibialis anterior muscle in patients with low back pain. Likewise, a statistically significant functionality improvement is seen for CG that received alternative treatment in meniscal injuries [22,46], SAIS [23] and knee OA $[42,43,48]$, although they did not show to be better than MF treatment. On the other hand, a decrease in pain is observed in favor of EG for VAS, KOOS, and NPRS, although with statistical significance only for strengthening MF in meniscal injuries and OA $(p<0.05)$ aimed at increasing quadriceps femoris strength (medial and lateral vastus) $[22,41-43,48]$. Gait speed shows an improvement in both groups between evaluation sessions, although without significant differences [22]. EMG-A increased in both study groups, although with greater significance $(p<0.05)$ in EG when using strengthening MF in participants with meniscal injury [22,35,46,47], SAIS [36], PFPS [37], ACLR [44], and knee OA [48].

The main muscles trained in knee conditions were quadriceps femoris, trapezius and anterior serratus in SAIS. On the other hand, 4 studies evaluated muscle strength changes in patients with knee OA showing an increase in both groups, although with significant differences $(p<0.05)$ in favor of MF groups in which the vastus medialis was trained $[39,40,42,43]$. On the other hand, Choi reported an increase in vastus medialis thickness in patients with knee OA with significant changes compared to CG $(p<0.05)$ [41]. Regarding ROM, an improvement is observed after MF treatment, although with controversial results, reporting statistical significance by Akkaya, Christanell, and Kirnap [22,44,46], and without differences between groups according to Draper and Yilmaz $[38,42]$. Although the decrease in edema was documented as a secondary outcome, only Christanell reported its changes, showing a subjective reduction in patients with ACLR [44].

\section{Muscle relaxation MF studies}

Main outcomes for muscle relaxation MF studies included functionality (disability) changes ( $\mathrm{n}=8,80.0 \%)$ $[24,25,49-51,53-55]$, pain intensity decrease $(n=7$, $70.0 \%) \quad[24,25,50,53-56]$, mental health improve $(\mathrm{n}=5,50 \%)[24,25,50,54,56]$, EMG-A $(\mathrm{n}=2,20.0 \%)$ [24,52], ROM changes $(\mathrm{n}=1,10.0 \%)$ [53], movement fear $(\mathrm{n}=1,10.0 \%)$ [25], muscle strength changes [50] and health state $(\mathrm{n}=1,10.0 \%)$ [24]. Functionality was assessed with the Canadian Occupational Performance Scale (COPM) [49,51], Psychosocial Functioning Scale (MPI-S) [49,51], Work Ability Index (WAI) [50], Purdue Pegboard Test (PPT) [26], FCI [24], ODI [25], neck disability index [53], pain disability index $[54,55]$ and general activity level scale (GALS) [54]. Pain intensity was assessed with NPRS [50], pain self-perception scale [24], pressure algometry (PA) [24,53], symptom checklist [24], VAS [25,53,55], pain beliefs personality questionnaire (PBQ) [54,56], pain self-report [54], and West Haven Yale multidimensional inventory (WHYMPI) [56]. On the other hand, mental health was assessed through participants' self-report [50], Beck's depression inventory (BDI) $[24,25,54,56]$ and state-trait anxiety inventory (STAI) [54]. EMG-A, ROM and movement fear were evaluated using surface electromiography, inclinometry, and Tampa scale for kinesiophobia (TSK) respectively $[25,52,53]$. Finally, muscle strength and health status were assessed through dynamometry and the SF-36 health questionnaire $[24,50]$.

Results show an improvement in functionality for EG and $\mathrm{CG}$, although with significance in favor of MF for COMP $(\mathrm{p}<0.01)$, MPI-S ( $<<0.016)$, WAI $(\mathrm{p}<0.01)$, NDI $(\mathrm{p}<0.01)$, and PDI $(\mathrm{p}<0.03)$ in participants with cervicobrachialgia [49,55], neck pain [50,53] and WPS [51], in whom the aim was to reduce the activity of the trapezius muscle. $\mathrm{CG}$ also shows an improvement in functionality $(\mathrm{p}<0.05)$ between the evaluation sessions for the COMP, MPI-S, and WAI, although without being better than MF [49-51]. A decrease in pain is observed in EG for NPRS [50], VAS [25,53], PA [24,53], PBQ [54], and pain self-report [54] in participants with neck pain, fibromyalgia, LBPS, although without statistical significance $(p>0.05)$. These studies show MF applications in the upper trapezius for cervical conditions and spinal erector for LBPS. Despite the above, Dellve (2011) reported a greater decrease in pain in patients with neck pain for one CG that did not receive treatment $(\mathrm{p}<0.046)$ [50]. Mental health shows improvements for both study groups, although Spence (1995) reports statistical significance in favor of controls for BDI in cervicobrachialgia $(p<0.01)$ [56]. EMG-A shows a decrease in patients with cervical radiculopathy in favor of MF $(\mathrm{p}<0.01)$ [52] and WPS $(\mathrm{p}<0.046)$ [25]. On the other hand, ROM increased for both groups in patients with neck pain highlights (intragroup changes) $(p<0.001)$ but without differences between them $(p>0.05)$. Finally, movement fear, muscle strength and health state evaluated do not show statistically significant intra - or intergroup changes $(p>0.05)$ [24,50,51].

\section{Discussion}

The objective of this SR was to investigate the scientific evidence on the MF efficacy in function recovery, strength increase, and muscle relaxation in MSD. Low risk of bias was assessed for most of the articles, showing only some concerns in the random assignment 
domain for the RoB2 tool $[22,23,37,39,40-51,53,55,56]$. PEDro score shows good internal validity for 17 studies (65.38\%) supporting the methodology and results of the analyzed articles [33].

The studies were classified in two therapeutic applications: strengthening $(\mathrm{n}=16,61.53 \%)$ and muscle relaxation $(\mathrm{n}=10,38.46 \%)$. Both applications are interesting because they support MF's ability to detect subtle changes in motor neurons' activity, often difficult to objectify with palpation or observation, in patients treated to increase (strengthen) or decrease (relaxation) muscle activity. It should be noted that MF systems are based on the information principle (Ross Ashby's law), in which a variable is correctly controlled (strength or relaxation) if the controller (patient) has enough information (visual and/or audible).

\section{Strengthening MF applications}

This review supports MF efficacy for strengthening in knee OA, meniscal injury, SAISS, PFPS, ACLR, and lumbar discopathy when it is complemented with therapeutic exercises, FES, NMES, lymphatic drainage, and superficial thermotherapy showing greater benefits than controls that received the same treatments, but without MF. MF applications focused on joint MSD that included knee conditions $(\mathrm{n}=13)$ [22,23,37-44,46-48], shoulder $(n=2)[23,36]$ and lumbar spine $(n=1)[45]$. Knee conditions included postsurgical $(n=10)$ and nonsurgical $(n=3)$ MF rehabilitation focusing on quadriceps femoris strength recovery. Quadriceps femoris strengthening in these disorders is key due to muscle inhibition caused by pain and joint inflammation (arthrogenic muscle inhibition, AMI). AMI results in an altered proprioceptive information decreasing quadriceps femoris strength, whose role is essential for lower limb functional activities [58].

MF stimulates neural circuits, providing new somatosensory information (visual and/or auditory) to motor activities, recovering afferent and proprioceptive information. Motor control is influenced by visual and auditory systems through motor pathways modulation (corticospinal pathway) that regulates A-alpha motor neurons. In this line, MF favors the activation of these systems by modulating motor activity at different levels of CNS $[29,30]$. On the other hand, shoulder and LBPS treatments included MF training in trapezius and serratus anterior, and tibialis anterior respectively $[23,36,45]$. MF can recover scapulothoracic muscles strength, whose imbalances lead to scapular dysfunctions in addition to biomechanical alterations, such as decreased subacromial space and scapular upward [59]. MF in shoulder rehabilitation protocols can be a valuable resource to correct muscle imbalances by providing patients with visual or auditory information to activate stabilizer scapular muscles while performing exercises. Likewise, it is suggested, for muscular imbalance management, to first use relaxation MF in facilitated muscles and later activation MF in inhibited muscles. This review shows the MF application in the tibialis anterior muscle reporting improvements in functionality in patients with LBPS [45]. Although this peripheral activation has not been clarified, facilitation of neurological circuits of lumbar spinal cord segments (L4L5), responsible for motor control at the lumbar level, is recommended. Peripheral activation could facilitate lumbar spinal segments motor neurons, also associated with lower back muscles so that an MF training could result in lumbar muscles indirect activation.

Strengthening MF offered increased functionality, decreased pain, increased ROM, and electromyographic activity. Functionality was tested with different validated instruments which support stable, safe, and consistent results for strengthening MF therapy: Lyshom scales, test-retest $($ TRT $)=0.91$ and internal consistency (IC) $=0.65 ; \mathrm{KOOS}, \mathrm{TRT}=0.87-0.96$ and $\mathrm{IC}=0.78 ; \mathrm{DASH}$, TRT $=0.97$ and $\mathrm{IC}=0.96$; OSS, TRT $=0.82-0.91$; ODI, TRT $=0.83-0.99$ and $\mathrm{IC}=0.71-0.87$; WOMAC, TRT $=0.83-0.90$ and $\mathrm{IC}=0.70-0.93$ [60-64]. This review shows an improvement in functionality, supporting the efficacy of strengthening MF in meniscal injuries, SAIS, and knee OA. Although it is complex to explain a direct relationship between MF and functionality, these changes could be the result of the visual and auditory integration, at different levels, of the CNS due to biofeedback, which allows patients to better control their motor activity as occurs with daily life tasks in which motor patterns are constantly fed back by the somatosensory and proprioceptive systems [65]. Functional improvements can also be explained by the "pain-fear-disability" circle interruption, a model that explains the close relationship between pain, its emotional factors, and consequent disability, and that would be modulated by visual or auditive stimuli when the patient trains with MF [66].

Attention and concentration are factors that influence pain perception, involving the participation of descending modulatory mechanisms whose antinociceptive effects may be equal to or better than those of morphine. This antinociceptive effect seem to be supported by the decrease in thalamocortical activity, thalamus, somatosensory areas, insula and anterior cingulate gyrus. The effect results in pain decrease perception when training with MF due to the patient's lessened attention to the injury while concentrating on the visual and auditory stimuli.

Other analgesic mechanisms have supported endogenous opioid peptides release with motor electrical stimulation [68]. MF allows a simultaneous NMES with MVC configuration while feedback is generated, which 
could favor endogenous opioids release when training with electrical currents; however, studies do not document combined applications of MF and NMES. On the other hand, MF motor pathways (different levels), visual, and auditory cortex activation modulate muscle tone by adjusting it to perform a certain motor activity, interrupting the muscle spasm-pain circle (muscle tone self-regulation) [69].

According to the results for strengthening MF, 15 to 20 sessions 3 to 5 times per week are recommended to ensure functionality improvements and muscle strength. Although treatment times are varied, it seems that there are sufficient interventions between 15 and 30 minutes.

It should be noted that, for MF training development, the patient must be able to perform voluntary muscle activity. This finding suggests that MF intervention for strengthening is more useful in MSD in which muscle function is preserved. In addition, it is important to explain to patients that the equipment is not the one that improves strength but only monitors their muscle activity.

\section{Relaxation MF applications}

Relaxation MF generally uses audible feedback while monitoring motor neuron activity $(\mu \mathrm{V})$, emitting a sound stimulus when electromyographic activity exceeds a preset baseline activity level, ensuring that the patient consciously inhibits their muscles. Despite the above, mixed biofeedback (visual and auditory) is seen in MF relaxation studies.

This review supports MF efficacy for muscle relaxation in neck pain, cervicobrachialgia, fibromyalgia, and LBPS when it is complemented with cervical distraction, superficial thermotherapy, US, and therapeutic exercises, showing better therapeutic effects than controls that received the same interventions without MF.

MF applications focused on non-specific MSP on the cervical spine and lumbar spine. The applications were on the trapezius muscle (upper portion) for cervical conditions and spinal erectors for participants with LBPS. The trapezius muscle is usually facilitated or appears more active in neck pain conditions due to the fact that it is responsible for dysfunctional postural patterns such as superior cruciate syndrome. A similar phenomenon occurs with lumbar erectors that are facilitated in low back pain conditions and that lead to postural dysfunctions, such as inferior crossed syndrome [70].

MF studies show improvements in functionality and pain decrease. Functionality was evaluated with validated instruments, which supports results for relaxation MF studies: ODI, TRT $=0.83-0.99$ and $\mathrm{IC}=0.71-0.87$; NDI TRT $=0.50-0.98$ and $\mathrm{IC}=0.85 ;$ WAI TRT $=0.92$ and $\mathrm{IC}=0.74[71,72]$.

Improvements in functionality can be explained by the "pain-fear-disability" circle interruption and muscle tone self-regulation (previously explained). This improvement can also be explained by the "information principle," in which the variable (muscle tone) is controlled when the person has information about it. On the other hand, selfregulation of muscle tone added to concentration on the task while training with MF could explain the analgesic effects, mediated by changes in attention [67].

It is interesting that some studies considered health status, movement fear, and depression as secondary outcomes, especially considering that MF showed benefits in patients with fibromyalgia and LBPS. These results support the MF central modulation that could exert an inhibitory effect at the limbic system, modifying psycho-affective response and pain perception of the individual [12].

According to the results, 8 to 12 sessions 3 to 5 times per week are suggested to achieve favorable functional changes for relaxation with MF. Although the treatment times are varied, interventions between 30 and $60 \mathrm{~min}$ utes are recommended. Likewise, it should be considered that, due to prolonged treatment time, many clinicians could opt for other relaxation strategies despite the clear benefits and comparative advantages that MF therapy shows.

\section{Conclusions}

MF is a safe and non-invasive treatment used in rehabilitation for different MSD. This treatment can be used for muscle strengthening (active method) or relaxation (passive method), although both techniques require muscle function indemnity.

This SR shows that strengthening and relaxation MF applications are effective for improving the functionality and reducing pain in the short and long term for different joint and soft tissue disorders, especially when complemented with therapeutic exercises or physical agents. MF treatment shows good results in psychoaffective variables such as movement fear and depression, suggesting a modulating influence on the CNS. Although it was not possible to perform a meta-analysis of the studies due to their heterogeneity, these results seem promising and promote MF in the rehabilitation protocols of the revised MSDs, as well as the development of new research on other musculoskeletal conditions not documented in this review.

Despite the results, a common aspect for MF applications is the time required to prepare the patient as well as the time needed to carry out the training, which could discourage its use despite its benefits. Likewise, this SR allowed the researchers to propose a dosage recommendation for strengthening and relaxation with MF, which can be revised and considered for future research. 


\section{Funding}

This research received no external funding.

\section{Conflict of interests}

The authors have no conflict of interest to declare.

\section{References}

1. Hawker GA. The assessment of musculoskeletal pain. Clin Exp Rheumatol. 2017; 35 Suppl 107(5): 8-12.

2. El-Tallawy SN, Nalamasu R, Salem GI, LeQuang JAK, Pergolizzi JV, Christo PJ. Management of musculoskeletal pain: An update with emphasis on chronic musculoskeletal pain. Pain Ther. 2021; 10(1): 181-209.

3. Booth J, Moseley GL, Schiltenwolf M, Cashin A, Davies $M$, Hübscher M. Exercise for chronic musculoskeletal pain: A biopsychosocial approach. Musculoskeletal Care. 2017; 15(4): 413-21.

4. Park PW, Dryer RD, Hegeman-Dingle R, Mardekian J, Zlateva G, Wolff GG, et al. Cost burden of chronic pain patients in a large integrated delivery system in the United States. Pain Pract. 2015; 16(8): 1001-11.

5. Arendt-Nielsen L, Fernández-de-Las-Peñas C, Graven-Nielsen T. Basic aspects of musculoskeletal pain: from acute to chronic pain. J Man Manip Ther. 2011; 19(4): 186-93.

6. Blyth FM, Noguchi N. Chronic musculoskeletal pain and its impact on older people. Best Pract Res Clin Rheumatol. 2017; 31(2): 160-8.

7. Jensen TS, Baron R, Haanpää M, Kalso E, Loeser JD, Rice ASC, et al. A new definition of neuropathic pain. Pain. 2011; 152(10): 2204-5.

8. Szok D, Tajti J, Nyári A, Vécsei L. Therapeutic approaches for peripheral and central neuropathic pain. Behav Neurol. 2019; 2019: 8685954.

9. van Griensven H, Schmid A, Trendafilova T, Low M. Central sensitization in musculoskeletal pain: Lost in translation? J Orthop Sports Phys Ther. 2020; 50(11): 592-6.

10. Pelletier R, Higgins J, Bourbonnais D. Is neuroplasticity in the central nervous system the missing link to our understanding of chronic musculoskeletal disorders? BMC Musculoskelet Disord. 2015; 16(1): 25.

11. Baliki MN, Mansour AR, Baria AT, Apkarian AV. Functional reorganization of the default mode network across chronic pain conditions. PLoS One. 2014; 9(9): e106133.

12. Fenton BW, Shih E, Zolton J. The neurobiology of pain perception in normal and persistent pain. Pain Manag. 2015; 5(4): 297-317.

13. Vos T, Flaxman AD, Naghavi M, Lozano R, Michaud C, Ezzati M, et al. Years lived with disability (YLDs) for 1160 sequelae of 289 diseases and injuries 1990 2010: a systematic analysis for the Global Burden of Disease Study 2010. Lancet. 2012; 380(9859): 216396.

14. GBD 2017 Disease and Injury Incidence and Prevalence Collaborators. Global, regional, and national incidence, prevalence, and years lived with disability for 354 diseases and injuries for 195 countries and territories, 1990-2017: a systematic analysis for the Global Burden of Disease Study 2017. Lancet. 2018; 392(10159): 1789-858.

15. Lin I, Wiles L, Waller R, Goucke R, Nagree Y, Gibberd $\mathrm{M}$, et al. What does best practice care for musculoskeletal pain look like? Eleven consistent recommendations from high-quality clinical practice guidelines: systematic review. Br J Sports Med. 2020; 54(2): 79-86.

16. Abdel Shaheed C, Maher CG, Williams KA, Day R, McLachlan AJ. Efficacy, tolerability, and dose-dependent effects of opioid analgesics for low back pain: A systematic review and meta-analysis: A systematic review and meta-analysis. JAMA Intern Med. 2016; 176(7): 958-68.

17. Mercadante S, Arcuri E, Santoni A. Opioid-induced tolerance and hyperalgesia. CNS Drugs. 2019; 33(10): 943-55.

18. Lin I, Wiles LK, Waller R, Goucke R, Nagree Y, Gibberd $\mathrm{M}$, et al. Poor overall quality of clinical practice guidelines for musculoskeletal pain: a systematic review. Br J Sports Med. 2018; 52(5): 337-43.

19. Beutler A. Musculoskeletal therapies: Adjunctive physical therapy. FP Essent. 2018; 470: 16-20.

20. Hidalgo B, Detrembleur C, Hall T, Mahaudens P, Nielens $H$. The efficacy of manual therapy and exercise for different stages of non-specific low back pain: an update of systematic reviews. J Man Manip Ther. 2014; 22(2): 59-74.

21. Smith BE, Hendrick P, Bateman M, Holden S, Littlewood C, Smith TO, et al. Musculoskeletal pain and exercise-challenging existing paradigms and introducing new. Br J Sports Med. 2019; 53(14): 907-12.

22. Akkaya N, Ardic F, Ozgen M, Akkaya S, Sahin F, Kilic A. Efficacy of electromyographic biofeedback and electrical stimulation following arthroscopic partial meniscectomy: a randomized controlled trial. Clin Rehabil. 2012; 26(3): 224-36.

23. Juul-Kristensen B, Larsen CM, Eshoj H, Clemmensen T, Hansen A, Bo Jensen P, et al. Positive effects of neuromuscular shoulder exercises with or without EMGbiofeedback, on pain and function in participants with subacromial pain syndrome - A randomised controlled trial. J Electromyogr Kinesiol. 2019; 48: 161-8.

24. Baumueller E, Winkelmann A, Irnich D, Weigl M. Electromyogram biofeedback in patients with 
fibromyalgia: A randomized controlled trial. Complement Med Res. 2017; 24(1): 33-9.

25. Gálvez-Hernández CL, Rodríguez-Ortiz MD, Del RíoPortilla Y. Biofeedback treatment for acute whiplash patients. Rev Med Inst Mex Seguro Soc. 2016; 54(4): 480-9.

26. Narayanan SP, Bharucha AE. A practical guide to biofeedback therapy for pelvic floor disorders. Curr Gastroenterol Rep. 2019; 21(5): 21.

27. Dost Sürücü G, Tezen Ö. The effect of EMG biofeedback on lower extremity functions in hemiplegic patients. Acta Neurol Belg. 2021; 121(1): 113-8.

28. Robinson AJ, Snyder-Mackler L, editors. Clinical Electrophysiology: Electrotherapy and Electrophysiologic Testing. 3rd ed. Philadelphia, PA: Lippincott Williams and Wilkins; 2007.

29. Avanzino L, Pelosin E, Vicario CM, Lagravinese G, Abbruzzese G, Martino D. Time processing and motor control in movement disorders. Front Hum Neurosci. 2016; 10: 631 .

30. Moher D, Liberati A, Tetzlaff J, Altman DG, PRISMA Group. Preferred reporting items for systematic reviews and meta-analyses: the PRISMA statement. BMJ. 2009; 339: b2535.

31. Ouzzani M, Hammady H, Fedorowicz Z, Elmagarmid A. Rayyan - a web and mobile app for systematic reviews. Syst Rev. 2016; 5(1).

32. Albanese E, Bütikofer L, Armijo-Olivo S, Ha C, Egger M. Construct validity of the Physiotherapy Evidence Database (PEDro) quality scale for randomized trials: Item response theory and factor analyses. Res Synth Methods. 2020; 11(2): 227-36.

33. Sterne JAC, Savović J, Page MJ, Elbers RG, Blencowe NS, Boutron I, et al. RoB 2: a revised tool for assessing risk of bias in randomised trials. BMJ. 2019; 366:1 4898.

34. McGuinness LA, Higgins JPT. Risk-of-bias VISualization (robvis): An R package and Shiny web app for visualizing risk-of-bias assessments. Res Synth Methods. 2021; 12(1): 55-61.

35. Oravitan M, Avram C. The effectiveness of electromyographic biofeedback as part of a meniscal repair rehabilitation programme. J Sports Sci Med. 2013; 12(3): 526-32.

36. Huang H-Y, Lin J-J, Guo YL, Wang WT-J, Chen Y-J. EMG biofeedback effectiveness to alter muscle activity pattern and scapular kinematics in subjects with and without shoulder impingement. J Electromyogr Kinesiol. 2013; 23(1): 267-74.

37. Ng GYF, Zhang AQ, Li CK. Biofeedback exercise improved the EMG activity ratio of the medial and lateral vasti muscles in subjects with patellofemoral pain syndrome. J Electromyogr Kinesiol. 2008; 18(1): 128-33.
38. Draper V, Ballard L. Electrical stimulation versus electromyographic biofeedback in the recovery of quadriceps femoris muscle function following anterior cruciate ligament surgery. Phys Ther. 1991; 71(6): 455-61.

39. Anwer S, Quddus N, Miraj M, Equebal A. Effectiveness of electromyographic biofeedback training on quadriceps muscle strength in osteoarthritis of knee. Hong Kong Physiother J. 2011; 29(2): 86-93.

40. Dursun N, Dursun E, Kiliç Z. Electromyographic biofeedback-controlled exercise versus conservative care for patellofemoral pain syndrome. Arch Phys Med Rehabil. 2001; 82(12): 1692-5.

41. Choi YL, Kim BK, Hwang YP, Moon OK, Choi WS. Effects of isometric exercise using biofeedback on maximum voluntary isometric contraction, pain, and muscle thickness in patients with knee osteoarthritis. J Phys Ther Sci. 2015; 27(1): 149-53.

42. Yilmaz OO, Senocak O, Sahin E, Baydar M, Gulbahar S, Bircan C, et al. Efficacy of EMG-biofeedback in knee osteoarthritis. Rheumatol Int. 2010; 30(7): 887-92.

43. Durmuş D, Alayli G, Cantürk F. Effects of quadriceps electrical stimulation program on clinical parameters in the patients with knee osteoarthritis. Clin Rheumatol. 2007; 26(5): 674-8.

44. Christanell F, Hoser C, Huber R, Fink C, Luomajoki $H$. The influence of electromyographic biofeedback therapy on knee extension following anterior cruciate ligament reconstruction: a randomized controlled trial. Sports Med Arthrosc Rehabil Ther Technol. 2012; 4(1): 41.

45. Sardaru DP, Matei D, Zaharia-Kezdi D, Pendefunda L. Effects of biofeedback versus switch-triggered functional electrical stimulation on sciatica-related foot drop. J Back Musculoskelet Rehabil. 2018; 31(2): 239-45.

46. Kirnap M, Calis M, Turgut AO, Halici M, Tuncel M. The efficacy of EMG-biofeedback training on quadriceps muscle strength in patients after arthroscopic meniscectomy. N Z Med J. 2005; 118(1224): U1704.

47. Levitt R, Deisinger JA, Remondet Wall J, Ford L, Cassisi JE. EMG feedback-assisted postoperative rehabilitation of minor arthroscopic knee surgeries. J Sports Med Phys Fitness. 1995; 35(3): 218-23.

48. Raeissadat SA, Rayegani SM, Sedighipour L, Bossaghzade Z, Abdollahzadeh MH, Nikray R, et al. The efficacy of electromyographic biofeedback on pain, function, and maximal thickness of vastus medialis oblique muscle in patients with knee osteoarthritis: a randomized clinical trial. J Pain Res. 2018; 11: 2781-9.

49. Ehrenborg C, Archenholtz B. Is surface EMG biofeedback an effective training method for persons with neck and shoulder complaints after whiplash- 
associated disorders concerning activities of daily living and pain - a randomized controlled trial. Clin Rehabil. 2010; 24(8): 715-26.

50. Dellve L, Ahlstrom L, Jonsson A, Sandsjö L, Forsman M, Lindegård A, et al. Myofeedback training and intensive muscular strength training to decrease pain and improve work ability among female workers on long-term sick leave with neck pain: a randomized controlled trial. Int Arch Occup Environ Health. 2011; 84(3): 335-46.

51. Voerman GE, Vollenbroek-Hutten MMR, Hermens HJ. Changes in pain, disability, and muscle activation patterns in chronic whiplash patients after ambulant myofeedback training. Clin J Pain. 2006; 22(7): 656-63

52. Atteya AA. Biofeedback traction versus conventional traction in cervical radiculopathy. Neurosciences (Riyadh). 2004; 9(2): 91-3.

53. Eslamian F, Jahanjoo F, Dolatkhah N, Pishgahi A, Pirani A. Relative effectiveness of electroacupuncture and biofeedback in the treatment of neck and upper back myofascial pain: A randomized clinical trial. Arch Phys Med Rehabil. 2020; 101(5): 770-80.

54. Newton-John TR, Spence SH, Schotte D. Cognitivebehavioural therapy versus EMG biofeedback in the treatment of chronic low back pain. Behav Res Ther. 1995; 33(6): 691-7.

55. Kosterink SM, Huis in 't Veld RMHA, Cagnie B, Hasenbring M, Vollenbroek-Hutten MMR. The clinical effectiveness of a myofeedback-based teletreatment service in patients with non-specific neck and shoulder pain: a randomized controlled trial. J Telemed Telecare. $2010 ; 16(6)$ : 316-21.

56. Spence SH, Sharpe L, Newton-John T, Champion D. Effect of EMG biofeedback compared to applied relaxation training with chronic, upper extremity cumulative trauma disorders. Pain. 1995; 63(2): 199-206.

57. Stovold E, Beecher D, Foxlee R, Noel-Storr A. Study flow diagrams in Cochrane systematic review updates: an adapted PRISMA flow diagram. Syst Rev. 2014; 3(1): 54 .

58. Sonnery-Cottet B, Saithna A, Quelard B, Daggett M, Borade A, Ouanezar H, et al. Arthrogenic muscle inhibition after ACL reconstruction: a scoping review of the efficacy of interventions. Br J Sports Med. 2019; 53(5): 289-98.

59. Struyf F, Nijs J, Mollekens S, Jeurissen I, Truijen S, Mottram S, et al. Scapular-focused treatment in patients with shoulder impingement syndrome: a randomized clinical trial. Clin Rheumatol. 2013; 32(1): $73-85$.

60. Kocher MS, Steadman JR, Briggs KK, Sterett WI, Hawkins RJ. Reliability, validity, and responsiveness of the Lysholm knee scale for various chondral disor- ders of the knee. J Bone Joint Surg Am. 2004; 86(6): 1139-45.

61. Ebrahimi N, Jalaie S, Salsabili N, Ansari NN, Naghdi S. Knee Injury and Osteoarthritis Outcome Score in patients with isolated meniscus injury; Validity and reliability. J Res Med Sci. 2017; 22(1): 55.

62. Raven EEJ, Haverkamp D, Sierevelt IN, van Montfoort DO, Pöll RG, Blankevoort L, et al. Construct validity and reliability of the disability of arm, shoulder, and hand questionnaire for upper extremity complaints in rheumatoid arthritis. J Rheumatol. 2008; 35(12): 2334-8.

63. van der Linde JA, van Kampen DA, van Beers LWAH, van Deurzen DFP, Terwee CB, Willems WJ. The Oxford Shoulder Instability Score; validation in Dutch and first-time assessment of its smallest detectable change. J Orthop Surg Res. 2015; 10(1): 146.

64. Roos EM, Toksvig-Larsen S. Knee injury and Osteoarthritis Outcome Score (KOOS) - validation and comparison to the WOMAC in total knee replacement. Health Qual Life Outcomes. 2003; 1(1): 17.

65. Cano-de-la-Cuerda R, Molero-Sánchez A, CarrataláTejada M, Alguacil-Diego IM, Molina-Rueda F, Miangolarra-Page JC, et al. Teorías y modelos de control y aprendizaje motor. Aplicaciones clínicas en neurorrehabilitación. Neurologia. 2015; 30(1): 32-41.

66. Zale EL, Lange KL, Fields SA, Ditre JW. The relation between pain-related fear and disability: a meta-analysis. J Pain. 2013; 14(10): 1019-30.

67. Roy M, Lebuis A, Peretz I, Rainville P. The modulation of pain by attention and emotion: a dissociation of perceptual and spinal nociceptive processes. Eur J Pain. 2011; 15(6): 641.e1-10.

68. Bi Y, Wei Z, Kong Y, Hu L. Supraspinal neural mechanisms of the analgesic effect produced by transcutaneous electrical nerve stimulation. Brain Struct Funct. 2021; 226(1): 151-62.

69. Johnson DS. The spasm-pain-spasm cycle. Pain Med. 2012; 13(4): 615.

70. Sembrano JN, Yson SC, Kanu OC, Braman JP, Santos ERG, Harrison AK, et al. Neck-shoulder crossover: how often do neck and shoulder pathology masquerade as each other? Am J Orthop (Belle Mead NJ). 2013; 42(9): E76-80.

71. MacDermid JC, Walton DM, Avery S, Blanchard A, Etruw E, McAlpine C, et al. Measurement properties of the neck disability index: a systematic review. J Orthop Sports Phys Ther. 2009; 39(5): 400-17.

72. de Zwart BCH, Frings-Dresen MHW, van Duivenbooden JC. Test-retest reliability of the Work Ability Index questionnaire. Occup Med (Lond). 2002; 52(4): 177-81. 